Article

\title{
Fiber Patterns in Young Adults Living in Different Environments (USA, Spain, and Tunisia). Anthropometric and Lifestyle Characteristics
}

\author{
María José García-Meseguer ${ }^{1, *}$, Amalia Delicado-Soria ${ }^{1}$ and Ramón Serrano-Urrea ${ }^{2}$ \\ 1 Department of Nursing, Physiotherapy and Occupational Therapy, Faculty of Nursing, University of \\ Castilla-La Mancha, Avda. de España, s/n, 02071 Albacete, Spain; amalia.delicado@gmail.com \\ 2 Department of Mathematics, Faculty of Computer Science Engineering, University of Castilla-La Mancha, \\ Avda. de España, s/n, 02071 Albacete, Spain; ramon.serrano@uclm.es \\ * Correspondence: mariajosefa.garcia@uclm.es; Tel.: +34-967-599-200
}

Received: 21 July 2017; Accepted: 13 September 2017; Published: 18 September 2017

\begin{abstract}
Benefits of dietary fiber go beyond its effect on chronic diseases associated with development. Consequently, the pattern of fiber intake has been considered an indicator for diet quality. Young adults are especially vulnerable to a food environment that drives an increase in chronic diseases linked to economic development. The aim of this work was to characterize patterns of fiber intake among university students. A cross-sectional study was conducted on a sample of 730 students enrolled at the University of Castilla-La Mancha (Spain), the University of Carthage (Tunisia), and Florida International University (USA). Mean age was 21.2. Food consumption was self-reported in two 24-h recalls. Mean dietary fiber intake was $17.8 \mathrm{~g}$, not reaching the adequate intake. Contrary to expectations, American participants were the highest consumers $(p<0.001)$, and also exhibited the highest BMI. Cereals, legumes, vegetables and fruit were the main food sources of fiber. Fiber from appetizers, prepared and precooked meals, sauces, spices and condiments accounted for $16.7 \%$ in American participants, $7.4 \%$ in Spanish participants and 2.6\% in Tunisian participants. Total fiber intake increased with energy intake but did not depend on smoking habits and physical activity in any country. It is essential to improve consumers' interpretation of guidelines on fiber intake.
\end{abstract}

Keywords: diet; fiber; socioeconomic factors; young adults; dietary pattern

\section{Introduction}

The association between fiber intake and health outcomes first came to public attention in the 1970 s, due to the detection of diseases in Western countries not existing on the African continent [1,2] such as diverticulitis, constipation or hemorrhoids. In this regard, reduction of fiber intake is inherent in changes from traditional food patterns to Western food models. This has led to an important change in recommendations on food and health, with an increase in daily fiber intake being actively promoted [3-5]. The key role of fiber intake in intestinal health is now well established [6,7], and it is also known that fiber-rich dietary patterns [8] and an adequate fiber intake is related to prevention of chronic diseases such as type 2 diabetes, obesity or certain types of cancer, whose prevalence is rising as a result of economic development and globalization [9-12]. Anthropometric and lifestyle characteristics were recently shown to mediate the association between fiber-rich dietary patterns and risk of chronic disease [8]. All generations are being affected by a food environment that favors the prevalence of these diseases, but young adults are especially vulnerable because they usually lead independent lifestyles and are unaware of other types of food environment [13].

In this scenario, and in order to improve the health state of people from middle and high-income countries, World Health Organization (WHO) and Food Agriculture Organization (FAO) policy 
guidelines $[14,15]$ include recommendations for fiber intake in countries' dietary guidelines. Therefore, patterns of fiber consumption may be considered an indicator of diet quality [16].

Although there is no universally accepted definition of fiber [17-19], definitions from the Institute of Medicine [20] are those most commonly found in the literature. Thus, total fiber is considered as the sum of dietary fiber and functional fiber. Moreover, it is known that different types of fiber may have different effects on health [21-24]. Since there is no recommended dietary allowance (RDA) or estimated average requirement (EAR), Adequate Intake (AI) has been defined a recommended average daily total fiber consumption of $14 \mathrm{~g} / 1000 \mathrm{Kcal}$ for adults of both genders [20].

Fiber structure has long been an area of interest, and a relationship has been established between fermentability, which is linked to chemical properties, and satiety and laxation [25-27]. Moreover, other positive effects of dietary fiber on chronic diseases have also been identified. These benefits are related to the complex function of fiber as a carrier of phytochemicals and its effect on gut microbiota [28-31]. Recent studies evidence that further knowledge of fiber healthy properties requires including information about food sources [32-34].

Despite country guidelines showing different fiber food sources according to the characteristics of the local diet, other factors such as price, taste, convenience or trends can strongly influence food choice, which will ultimately affect daily total fiber intake. This is the case of young adults. This population group presents an important challenge due to the coincidence of a series of emotional, physiological and environmental changes. They are usually sensitive to the influence of trends, which includes the consumption of soft drinks, snacks, prepared and pre-cooked meals and other ready-to-eat products, most of which are rich in sugars and fats, and deficient in fiber [35-37]. The transition to an independent life can be very stressful and can influence food choice, especially if they are living away from their parents' home and have poor cooking skills [38-41]. Epidemiological studies on this population group have shown that symptoms of diseases of affluence occur earlier than expected by biological age alone [42]. Thus, learning healthy food habits such as reaching an adequate fiber intake at an early age is critical for improving future health $[43,44]$. This is particularly important in university students, who are considered an important target group to promote healthy lifestyles [45].

To date, most studies have investigated food patterns of a country's overall population and the relationships with different diseases. Research focusing on young adults is, however, still limited. Although some studies on fiber intake have been conducted in young adults living in countries with different food patterns and incomes [33,42,45-48], to the best of our knowledge, no study has simultaneously assessed diet quality by fiber patterns in young people with homogeneous demographic and education characteristics but living in different environments (social, geographic, cultural, and economic). Knowledge of food patterns and fiber intake in young people from different countries, and the degree to which recommendations are followed, can help understand the complex relationships between young adults' behavior and food in different contexts.

Tunisia, Spain and the USA have different levels of development and different socio-cultural characteristics that directly influence their food patterns. In Tunisia, a low-middle income country [49], fiber consumption is decreasing mainly in urban areas, while the prevalence of chronic diseases inherent in globalization is rising [50,51]. Spain, a country where the Mediterranean food pattern is becoming less common, also presents lower total fiber consumption than recommendations [52]. In the United States, a Western model, guidelines promote strategies to increase fiber intake to improve American people's health [53,54]. Recent research in these countries has shown a decrease in fiber consumption among young adults relative to adult and older adult age groups [55-57].

The aim of this work was to characterize fiber consumption patterns of students at Florida International University (USA), the University of Castilla-La Mancha (Spain) and the University of Carthage (Tunisia), and to identify potential determinant factors. 


\section{Materials and Methods}

\subsection{Study Design}

A cross-sectional survey was administered to students enrolled at the University of Castilla-La Mancha, campus of Albacete, Spain (hereafter UCLM), the University of Carthage, Tunisian Republic (hereafter UCA) and Florida International University, Miami, FL, USA (hereafter FIU) during 2013. All procedures were in accordance with the WMA Declaration of Helsinki (Ethical Principles for Medical Research Involving Human Subjects). Informed consent was provided by each participant. The projects were approved by the following Ethics Committees: "Food habits of college students: University of Castilla-La Mancha (Albacete Campus)", approved by the Ethics Committee at the University Hospital Complex of Albacete (CEIC), Act No. 02/13, January 29, 2013; "Food habits of college students: Florida International University", approved by CEIC, Act No. 10/13, October 28, 2013, and FIU Institutional Review Board, IRB-13-0231, June 26, 2013; and "Food habits in a university population: Tunis Virtual University and the University of November 7 at Carthage (Tunisia)" approved by CEIC, Act No. 11/13, November 25, 2013.

\subsection{Study Participants}

Subjects were recruited using stratified sampling according to students enrolled on each degree course at the three campuses. Inclusion criteria were: (1) being enrolled at one of the three universities during 2013; (2) being aged between 18 and 30 years; (3) voluntarily agreeing to participate in the erratumsurvey; and (4) accepting and signing the informed consent. Exclusion criteria were: (1) not completing all the data in the surveys; and (2) presence of acute diseases (affecting diet) when the surveys were administered. In addition, an exclusion limit criterion was established, following the recommended intakes: (3) males whose daily energy intake was higher than $4000 \mathrm{Kcal} /$ day and less than $800 \mathrm{Kcal} /$ day, and females whose daily energy intake was higher than $3500 \mathrm{Kcal} /$ day and less than $500 \mathrm{Kcal} /$ day [58].

\subsection{General Data}

General information was self-reported by each subject using a questionnaire including the following items: (1) demographic data: gender, age; (2) anthropometric measurements: weight, height; (3) weight-loss diet: yes/no and (4) smoking habits: non smoker, $\leq 5$ cigarettes/day and $>5$ cigarettes/day. Body mass index (BMI) $\left(\mathrm{Kg} / \mathrm{m}^{2}\right)$ was calculated from anthropometric data and individuals were classified into four categories: underweight (BMI < 18.5), normal range $(18.5 \leq \mathrm{BMI} \leq 24.9)$, overweight $(25 \leq \mathrm{BMI} \leq 29.9)$ and obese $(\mathrm{BMI} \geq 30)[59]$.

\subsection{Food Consumption Assessment}

Food consumption data was gathered by two non-consecutive 24-h recalls including one weekend day. All food and beverages consumed were recorded by the recruited individuals. Trained dietary nurses explained how to complete the questionnaires, administered them and checked the data recorded. To help estimate portion sizes, participants were shown images of household measures and a Visual Guide [60,61].

Dial program 3.3.5 (Alceingenieria, Madrid, Spain) was used to determine energy and nutrients intake. Physical Activity Level (PAL) was calculated as the ratio of total to basal daily energy expenditure and classified individuals as: sedentary $(1.0 \leq \mathrm{PAL}<1.4)$, low active $(1.4 \leq \mathrm{PAL}<1.6)$, active $(1.6 \leq \mathrm{PAL}<1.9)$ and very active $(1.9 \leq \mathrm{PAL}<2.5)$ [62].

Daily fiber intake was calculated for each subject. The Dial program predetermines the following food group classification: cereals, legumes, vegetables, fruits and nuts, dairy products, meat, fish, eggs, sugar, sweets and pastries, fat and oil, non-dairy beverages, prepared and precooked meals, snacks, sauces, spices and condiments. Across these groups, all the foods that contributed to total fiber intake were reflected. 
The assessment was carried out using the following references: Tables and Dietary References of the Institute of Medicine [20], Tunisian and Spanish Food Composition Tables [63,64], and USDA Nutrient Database [65].

Taking into account the AI (14 g/1000 Kcal for both genders [20]), various approaches have been utilized in order to evaluate or define a level for a diagnosis of risk of insufficient fiber intake. Of these, a value of two thirds of AI has been accepted in different studies [66,67]. In our study, we calculated the percentage of students consuming less than two thirds of $\mathrm{AI}$, those whose intakes were more than two thirds of AI but less than AI, and those who reached AI.

\subsection{Statistical Analysis}

Statistical analysis was performed with IBM SPSS 22 (SPSS Inc., Chicago, IL, USA). Medians (and interquartile ranges) and means (and standard deviations) were used as descriptive statistics for quantitative variables. Proportions were used to describe qualitative variables. Kolmogorov-Smirnov and Shapiro-Wilk tests were used to study the normality of the distributions. The student's $t$ test (with previous Levene's test for equality of variances) and the Mann-Whitney $U$ test were used to compare two independent samples. The Kruskal-Wallis test (and Dunn post hoc method) were performed to compare more than two independent samples. When appropriate, trend analysis was performed using the Jonckheere-Terpstra trend test. The Chi-square test $\left(\chi^{2}\right)$ and the likelihood ratio test were used to compare proportions. Correlations were evaluated according to the Spearman's correlation coefficient. Level of significance was established as a $p$-value $<0.05$.

\section{Results}

A total of 775 students meeting inclusion criteria completed the surveys, providing all the required information. Nine were excluded due to the presence of diseases and 36 more individuals were excluded, as they did not meet daily energy intake limits. Therefore, the final sample consisted of 730 students (272 students from UCLM, 132 from UCA and 326 from FIU).

Mean age was 21.2 years, and gender distribution was 491 females and 239 males. It was a non-obese population (only $6.3 \%$ of the students showed obesity), whose mean BMI was $22.9 \mathrm{Kg} / \mathrm{m}^{2}$. Most of the students reported they were not smokers (88.1\%) and followed no weight-loss diets $(86.0 \%)$. Regarding the level of physical activity, $63.0 \%$ of the students were found to be active or very active. Mean daily energy intake was around $2000 \mathrm{Kcal}$ and mean daily total fiber intake was around $18 \mathrm{~g}$. We found statistically significant differences between females and males: energy intake was higher in males, and the percentages of overweight and obese students, as well as the percentage of smokers, were also higher in males (Table 1). BMI, total fiber intake and energy intake differences between females and males are shown in Table 2. It is interesting to note that only one fifth of the students at FIU classified as overweight or obese were following a diet when surveyed. Gender distribution showed that the percentage of American male students whose BMI was above the normal range was significantly higher than the percentage found in females ( $47.9 \%$ vs. $25.7 \%)$. Complete information about sociodemographic, anthropometric and lifestyle characteristics by country and gender are reported in Tables S1-S3 in supplementary material.

Table 1. Sociodemographic, anthropometric and lifestyle characteristics of the subjects (total sample).

\begin{tabular}{|c|c|c|c|c|}
\hline & Total Sample $(n=730)$ & Men $(n=239)$ & Women $(n=491)$ & $p$ \\
\hline Population (\%) & 100 & 32.7 & 67.3 & - \\
\hline \multicolumn{5}{|l|}{ Age (years) } \\
\hline Mean + SD $(95 \%$ CI $)$ & $21.2 \pm 2.8(21.0-21.4)$ & $21.2 \pm 2.9(20.9-21.6)$ & $21.2 \pm 2.7(21.0-21.5)$ & $p=0.705^{\dagger}$ \\
\hline Median (IR) & $21(4)$ & $20(4)$ & $21(3)$ & \\
\hline \multicolumn{5}{|l|}{ Weight $(\mathrm{Kg})$} \\
\hline Mean + SD $(95 \%$ CI $)$ & $65.0 \pm 13.3(64.0-65.9)$ & $75.7 \pm 12.3(74.1-77.2)$ & $59.8 \pm 10.3(58.9-60.7)$ & $p<0.001^{t, *}$ \\
\hline Median (IR) & $63(17.0)$ & $74.0(16.0)$ & $59.0(12.0)$ & \\
\hline
\end{tabular}


Table 1. Cont.

\begin{tabular}{|c|c|c|c|c|}
\hline & Total Sample $(n=730)$ & Men $(n=239)$ & Women $(n=491)$ & $p$ \\
\hline $\begin{array}{l}\text { BMI }\left(\mathrm{Kg} / \mathrm{m}^{2}\right) \\
\quad \text { Mean + SD }(95 \% \mathrm{CI}) \\
\quad \text { Median }(\mathrm{IR})\end{array}$ & $\begin{array}{c}22.9 \pm 13.3(22.6-23.2) \\
22.3(4.4)\end{array}$ & $\begin{array}{c}23.7 \pm 4.1(23.2-24.3) \\
23.3(4.3)\end{array}$ & $\begin{array}{c}22.4 \pm 4.0(22.1-22.8) \\
21.8(4.2)\end{array}$ & $p<0.001^{t, *}$ \\
\hline $\begin{array}{l}\text { BMI-categories (\%) } \\
\text { Underweight } \\
\text { Normal range } \\
\text { Overweight } \\
\text { Obese }\end{array}$ & $\begin{array}{c}8.2 \\
69.6 \\
15.9 \\
6.3\end{array}$ & $\begin{array}{c}6.7 \\
64.4 \\
19.2 \\
9.6\end{array}$ & $\begin{array}{c}9.0 \\
72.1 \\
14.3 \\
4.7\end{array}$ & $p=0.011 \S, *$ \\
\hline $\begin{array}{l}\text { BMI-2-categories (\%) } \\
\text { Underw. + normal rg. } \\
\text { Overweight + obese }\end{array}$ & $\begin{array}{l}77.8 \\
22.2 \\
\end{array}$ & $\begin{array}{l}71.1 \\
28.9\end{array}$ & $\begin{array}{l}81.1 \\
18.9\end{array}$ & $p=0.002 \S, *$ \\
\hline $\begin{array}{l}\text { Total fiber intake }(\mathrm{g}) \\
\text { Mean + SD }(95 \% \mathrm{CI}) \\
\text { Median (IR) }\end{array}$ & $\begin{array}{c}17.8 \pm 9.6(17.1-18.5) \\
15.6(8.6)\end{array}$ & $\begin{array}{c}17.2 \pm 8.4(16.2-18.3) \\
14.9(8.3)\end{array}$ & $\begin{array}{c}18.1 \pm 10.1(17.2-19.0) \\
15.7(9.0)\end{array}$ & $p=0.303^{\dagger}$ \\
\hline $\begin{array}{l}\text { Energy intake (Kcal/day) } \\
\text { Mean + SD } \\
(95 \% \text { CI) } \\
\text { Median (IR) }\end{array}$ & $\begin{array}{c}1971.9 \pm 553.0 \\
(1931.8-2012.1) \\
1932.5(747.9)\end{array}$ & $\begin{array}{c}2070.2 \pm 604.9 \\
(1993.1-2147.3) \\
2041.0(897.6)\end{array}$ & $\begin{array}{c}1924.1 \pm 519.9 \\
(1878.0-1970.2) \\
1889.0(730.0)\end{array}$ & $p=0.001^{\ddagger}, *$ \\
\hline Weight-loss diet (\%) & 14.0 & 14.6 & 13.6 & $p=0.715 \S$ \\
\hline $\begin{array}{l}\text { Smoking habits }(\%) \\
\text { Non-smoker } \\
\leq 5 \text { cigarettes per day } \\
>5 \text { cigarettes per day }\end{array}$ & $\begin{array}{l}88.1 \\
6.3 \\
5.6\end{array}$ & $\begin{array}{c}83.7 \\
7.9 \\
8.4\end{array}$ & $\begin{array}{c}90.2 \\
5.5 \\
4.3\end{array}$ & $p=0.029 \S, *$ \\
\hline $\begin{array}{l}\text { Physical activity }(\%) \\
\text { Sedentary } \\
\text { Low active } \\
\text { Active } \\
\text { Very active }\end{array}$ & $\begin{array}{c}9.0 \\
27.9 \\
40.3 \\
22.7\end{array}$ & $\begin{array}{c}7.5 \\
28.5 \\
32.6 \\
31.4\end{array}$ & $\begin{array}{c}9.8 \\
27.7 \\
44.0 \\
18.5\end{array}$ & $p<0.001 \S, *$ \\
\hline
\end{tabular}

SD: Standard deviation; CI: Confidence interval; IR: Interquartile range; BMI: Body mass index. ${ }^{\dagger}$ Mann-Whitney U test; ${ }^{\ddagger}$ Student's $t$ test; ${ }^{\circledR} \chi^{2}$ test; ${ }^{*}$ Significant differences.

Table 2. Body mass index (BMI), fiber intake and energy intake characteristics of the sample by universities.

\begin{tabular}{|c|c|c|c|c|}
\hline UCLM (SPAIN) & & & & \\
\hline & Total Sample $(n=272)$ & Men $(n=120)$ & Women $(n=152)$ & $p$ \\
\hline Population (\%) & 100 & 44.12 & 55.88 & - \\
\hline \multicolumn{5}{|l|}{ BMI $\left(\mathrm{Kg} / \mathrm{m}^{2}\right)$} \\
\hline Mean $+\mathrm{SD}(95 \% \mathrm{CI})$ & $22.4 \pm 3.3(22.0-22.8)$ & $22.8 \pm 3.4(22.2-23.4)$ & $22.1 \pm 3.2(21.5-22.6)$ & $p=0.041^{+, *}$ \\
\hline Median (IR) & $22.0(4.1)$ & $22.7(4.2)$ & $21.6(3.8)$ & \\
\hline \multicolumn{5}{|l|}{ BMI-categories (\%) } \\
\hline Underweight & 8.5 & 10 & 7.2 & \\
\hline Normal range & 73.2 & 67.5 & 77.7 & $p=0.297 \S \S$ \\
\hline Overweight & 14.7 & 17.5 & 12.5 & \\
\hline Obese & 3.7 & 5.0 & 2.6 & \\
\hline \multicolumn{5}{|l|}{ BMI-2-categories (\%) } \\
\hline Underw. + normal rg. & 81.7 & 77.5 & 84.9 & $p=0.119 \S$ \\
\hline Overweight + obese & 18.4 & 22.5 & 15.1 & \\
\hline \multicolumn{5}{|l|}{ Total fiber intake (g) } \\
\hline Mean $+\mathrm{SD}(95 \% \mathrm{CI})$ & $15.7 \pm 6.6(14.9-16.5)$ & $16.4 \pm 6.3(15.3-17.5)$ & $15.1 \pm 6.9(14.0-16.2)$ & $p=0.047^{\dagger, *}$ \\
\hline Median (IR) & $14.4(7.4)$ & $14.7(8.4)$ & $13.9(7.0)$ & \\
\hline \multicolumn{5}{|l|}{ Energy intake (Kcal/day) } \\
\hline Mean + SD & $1980.5 \pm 519.5$ & $2123.4 \pm 548.8$ & $1867.7 \pm 466.8$ & \\
\hline$(95 \%$ CI $)$ & (1918.5-2042.6) & $(2024.2-2222.6)$ & (1792.9-1942.5) & $p<0.001 \ddagger$,* \\
\hline Median (IR) & $1944.2(643.5)$ & $2067.6(729.3)$ & $1829.9(652.0)$ & \\
\hline \multicolumn{5}{|l|}{ UCA (TUNISIA) } \\
\hline & Total Sample $(n=132)$ & Men $(n=46)$ & Women $(n=86)$ & $p$ \\
\hline Population (\%) & 100 & 34.84 & 65.15 & \\
\hline \multicolumn{5}{|l|}{ BMI $\left(\mathrm{Kg} / \mathrm{m}^{2}\right)$} \\
\hline Mean + SD $(95 \%$ CI $)$ & $21.4 \pm 2.8(20.9-21.8)$ & $22.3 \pm 2.8(21.5-23.2)$ & $20.8 \pm 2.7(20.2-21.4)$ & $p=0.001^{t, *}$ \\
\hline Median (IR) & $21.1(3.6)$ & $22.2(3.7)$ & $20.6(4.0)$ & \\
\hline \multicolumn{5}{|l|}{ BMI-categories (\%) } \\
\hline Underweight & 15.9 & 6.5 & 20.9 & \\
\hline Normal range & 75.0 & 78.3 & 73.2 & $p=0.059 \S \S$ \\
\hline Overweight & 7.6 & 13.0 & 4.7 & \\
\hline Obese & 1.5 & 2.2 & 1.2 & \\
\hline
\end{tabular}


Table 2. Cont.

\begin{tabular}{|c|c|c|c|c|}
\hline \multicolumn{5}{|l|}{ UCA (TUNISIA) } \\
\hline & Total Sample $(n=132)$ & $\operatorname{Men}(n=46)$ & Women $(n=86)$ & $p$ \\
\hline \multicolumn{5}{|l|}{ BMI-2-categories (\%) } \\
\hline Underw. + normal rg. & 90.9 & 84.8 & 94.1 & \multirow{2}{*}{$p=0.073 \S$} \\
\hline Overweight + obese & 9.1 & 15.2 & 5.9 & \\
\hline \multicolumn{5}{|l|}{ Total fiber intake (g) } \\
\hline Mean + SD $(95 \%$ CI $)$ & $15.0 \pm 4.8(14.1-15.8)$ & $14.6 \pm 5.6(13.0-16.3)$ & $15.1 \pm 4.3(14.2-16.1)$ & \multirow{2}{*}{$p=0.352^{+}$} \\
\hline Median (IR) & $14.5(5.4)$ & $14.0(5.7)$ & $14.8(5.4)$ & \\
\hline \multicolumn{5}{|l|}{ Energy intake (Kcal/day) } \\
\hline Mean + SD & $1843.4 \pm 563.1$ & $1841.2 \pm 670.5$ & $1844.5 \pm 500.6$ & \multirow{2}{*}{$p=0.567^{\dagger}$} \\
\hline $\begin{array}{l}(95 \% \text { CI }) \\
\text { Median (IR) }\end{array}$ & $\begin{array}{c}(1746.4-1940.3) \\
1769.5(667.0)\end{array}$ & $\begin{array}{c}(1642.1-2040.4) \\
1672.5(786.0)\end{array}$ & $\begin{array}{c}(1737.2-1951.9) \\
1801.0(646.3)\end{array}$ & \\
\hline \multicolumn{5}{|l|}{ FIU (USA) } \\
\hline & Total Sample $(n=326)$ & Men $(n=73)$ & Women $(n=253)$ & $p$ \\
\hline Population (\%) & 100 & 22.39 & 77.61 & - \\
\hline \multicolumn{5}{|l|}{ BMI $\left(\mathrm{Kg} / \mathrm{m}^{2}\right)$} \\
\hline Mean + SD $(95 \%$ CI $)$ & $23.9 \pm 4.8(23.4-24.4)$ & $26.2 \pm 4.9(25.1-27.4)$ & $23.2 \pm 4.6(22.6-23.8)$ & \multirow{2}{*}{$p<0.001^{t, *}$} \\
\hline Median (IR) & $23.1(5.1)$ & $24.9(5.9)$ & $22.5(4.9)$ & \\
\hline \multicolumn{5}{|l|}{ BMI-categories (\%) } \\
\hline Underweight & 4.9 & 1.4 & 5.9 & \multirow{4}{*}{$p=0.001 \S \S, *$} \\
\hline Normal range & 64.4 & 50.7 & 68.4 & \\
\hline Overweight & 20.2 & 26.0 & 18.6 & \\
\hline Obese & 10.4 & 21.9 & 7.1 & \\
\hline \multicolumn{5}{|l|}{ BMI-2-categories (\%) } \\
\hline Underw. + normal rg. & 69.3 & 52.1 & 74.3 & \multirow{2}{*}{$p<0.001 \S, *$} \\
\hline Overweight + obese & 30.6 & 47.9 & 25.7 & \\
\hline \multicolumn{5}{|l|}{ Total fiber intake $(\mathrm{g})$} \\
\hline Mean $+\mathrm{SD}(95 \% \mathrm{CI})$ & $20.8 \pm 12.0(17.5-22.1)$ & $20.2 \pm 11.6(17.5-22.9)$ & $20.9 \pm 12.1(19.4-22.4)$ & \multirow[t]{2}{*}{$p=0.393^{\dagger}$} \\
\hline Median (IR) & $18.1(12.8)$ & $17.3(14.8)$ & $18.1(12.5)$ & \\
\hline \multicolumn{5}{|l|}{ Energy intake (Kcal/day) } \\
\hline Mean + SD & $2016.8 \pm 596.6$ & $2127.0 \pm 623.8$ & $1985.0 \pm 550.2$ & \multirow{3}{*}{$p=0.060 \ddagger$} \\
\hline$(95 \% \mathrm{CI})$ & $(1954.7-2078.9)$ & (1981.5-2272.6) & (1916.9-2053.1) & \\
\hline Median (IR) & $1993.0(841.5)$ & $2211.0(947.5)$ & $1947.0(808.0)$ & \\
\hline
\end{tabular}

Cross-country analysis showed different lifestyle habits (Table 3). Most of the variables studied showed significant differences between the students from the three universities: total energy intake, total fiber intake, tobacco consumption, physical activity, and weight-loss diets. The BMI variable also showed significant differences between the universities. Our results showed that the percentage of students at UCLM classified as overweight or obese (18.4\%) was more than twice the percentage of overweight or obese students at UCA (9.1\%). This percentage was even higher in American participants (30.6\%), being more than three times higher than that of Tunisian students.

Physical activity also has an important role in a healthy lifestyle. Although the overall sample showed a population consisting mainly of active or very active students, significant differences were found when the three countries were studied separately. While the percentage of sedentary or low active Spanish students reached almost $70 \%$, this percentage was only $28 \%$ in Tunisian students and barely $13.5 \%$ in the American sample.

Regarding daily fiber consumption, Table 3 shows significant differences between the students from FIU and students from UCA and UCLM. American participants presented the highest intakes, reaching mean and median values of around $20 \mathrm{~g} /$ day, while these values were only around $15 \mathrm{~g} /$ day in students from Mediterranean countries. As expected, according to the results obtained for total daily fiber intake, we found significant differences for fiber intake adjusted by energy between Mediterranean and American participants. 
Table 3. Sociodemographic, anthropometric, and lifestyle differences among the three populations.

\begin{tabular}{|c|c|c|c|c|c|c|c|c|c|c|c|c|c|}
\hline & \multicolumn{4}{|c|}{ UCLM (SPAIN) } & \multicolumn{4}{|c|}{ UCA (TUNISIA) } & \multicolumn{4}{|c|}{ FIU (USA) } & \multirow[t]{2}{*}{$p$} \\
\hline & Mean + SD & CI 95\% & Median & IR & Mean + SD & CI 95\% & Median & IR & Mean + SD & CI 95\% & Median & IR & \\
\hline Age (years) & $20.3 \pm 2.4$ & $20.0-20.5$ & $19.0^{\mathrm{a}}$ & 2 & $19.8 \pm 1.4$ & $19.6-20.1$ & $19.0^{\mathrm{a}}$ & 1 & $22.6 \pm 2.8$ & $22.3-22.9$ & $22.0^{\mathrm{a}, \mathrm{b}}$ & 3 & $p<0.001^{t, *}$ \\
\hline Weight (Kg) & $65.7 \pm 12.9$ & $64.2-67.3$ & 64 & 18.0 & $64.3 \pm 11.3$ & $62.4-66.3$ & 63.0 & 14.0 & $64.6 \pm 14.3$ & $63.0-66.2$ & 61.5 & 18.0 & $p=0.278^{+}$ \\
\hline BMI $\left(\mathrm{Kg} / \mathrm{m}^{2}\right)$ & $22.4 \pm 3.3$ & $22.0-22.8$ & $22^{c, d}$ & 4.1 & $21.4 \pm 2.8$ & $20.9-21.8$ & $21.1^{c, e}$ & 3.6 & $23.9 \pm 4.8$ & $23.4-24.4$ & $23.1^{\mathrm{d}, \mathrm{e}}$ & 5.1 & $p<0.001^{t, *}$ \\
\hline Total fiber intake (g) & $15.7 \pm 6.6$ & $14.9-16.5$ & $14.4^{\mathrm{f}}$ & 7.4 & $15.0 \pm 4.8$ & $14.1-15.8$ & $14.5^{\mathrm{g}}$ & 5.4 & $20.8 \pm 12.0$ & $19.5-22.1$ & $18.1^{\mathrm{f}, \mathrm{g}}$ & 12.8 & $p<0.001^{t, *}$ \\
\hline Fiber intake (g/1000 Kcal) & $8.1 \pm 3.2$ & $7.7-8.5$ & $7.5^{\mathrm{h}}$ & 3.4 & $8.4 \pm 2.2$ & $8.0-8.7$ & $8.0^{\mathrm{i}}$ & 2.7 & $10.5 \pm 5.6$ & $9.9-11.1$ & $8.9^{\mathrm{h}, \mathrm{i}}$ & 5.9 & $p<0.001^{t, *}$ \\
\hline Energy (Kcal/day) & $1980.5 \pm 519.5$ & $1918.5-2042.6$ & $1944.2^{j}$ & 643.5 & $1843.4 \pm 563.1$ & 1746.4-1940.3 & $1769.5^{\mathrm{j}, \mathrm{k}}$ & 667.0 & $2016.8 \pm 596.6$ & $1954.7-2078.9$ & $1993.0^{\mathrm{k}}$ & 841.5 & $p=0.002^{t, *}$ \\
\hline \multicolumn{14}{|l|}{ Weight-loss diet (\%) } \\
\hline Yes & \multirow{2}{*}{\multicolumn{4}{|c|}{$\begin{array}{c}5.1 \\
94.9\end{array}$}} & \multirow{2}{*}{\multicolumn{4}{|c|}{$\begin{array}{l}12.1 \\
87.9\end{array}$}} & \multirow{2}{*}{\multicolumn{4}{|c|}{$\begin{array}{l}22.1 \\
77.9\end{array}$}} & \multirow[t]{2}{*}{$p<0.001 \S, *$} \\
\hline No & & & & & & & & & & & & & \\
\hline \multicolumn{14}{|l|}{ Smoking habits (\%) } \\
\hline Non-smoker & \multicolumn{4}{|c|}{84.6} & \multicolumn{4}{|c|}{77.3} & \multicolumn{4}{|c|}{95.4} & \multirow{3}{*}{$p<0.001 \S \S, *$} \\
\hline$\leq 5$ cigarettes $/$ day & \multirow{2}{*}{\multicolumn{4}{|c|}{$\begin{array}{l}8.5 \\
7.0\end{array}$}} & \multirow{2}{*}{\multicolumn{4}{|c|}{$\begin{array}{c}8.3 \\
14.4\end{array}$}} & \multirow{2}{*}{\multicolumn{4}{|c|}{$\begin{array}{l}3.7 \\
0.9\end{array}$}} & \\
\hline$>5$ cigarettes/day & & & & & & & & & & & & & \\
\hline \multicolumn{14}{|l|}{ Level of physical activity (\%) } \\
\hline Sedentary & \multirow{2}{*}{\multicolumn{4}{|c|}{$\begin{array}{l}22.1 \\
47.4\end{array}$}} & \multirow{2}{*}{\multicolumn{4}{|c|}{$\begin{array}{c}1.5 \\
26.5\end{array}$}} & \multicolumn{4}{|c|}{1.2} & \multirow{4}{*}{$p<0.001 \S \S, *$} \\
\hline Low active & & & & & & & & & \multirow{2}{*}{\multicolumn{4}{|c|}{12.3}} & \\
\hline Active & \multirow{2}{*}{\multicolumn{4}{|c|}{$\begin{array}{l}20.2 \\
10.3\end{array}$}} & \multicolumn{4}{|c|}{43.2} & \multirow{2}{*}{\multicolumn{4}{|c|}{$\begin{array}{l}55.8 \\
30.7\end{array}$}} & \\
\hline Very active & & & & & \multicolumn{4}{|c|}{28.8} & & & & & \\
\hline \multicolumn{14}{|l|}{ BMI-categories (\%) } \\
\hline Underweight & & & & & & 15.9 & & & & 4.9 & & & \\
\hline Normal range & & 73.2 & & & & 75.0 & & & & 64.4 & & & $p<0.001 \$ \&, *$ \\
\hline Overweight & & 14.6 & & & & 7.6 & & & & 20.2 & & & \\
\hline Obese & & 3.7 & & & & 1.5 & & & & 10.4 & & & \\
\hline BMI-2-categories (\%) & & & & & & & & & & & & & \\
\hline Underw + normal & & 81.7 & & & & 90.9 & & & & 69.3 & & & $\mathrm{p}<0.001 \S_{,}^{*}$ \\
\hline Overw. + obese & & 18.3 & & & & 9.1 & & & & 30.6 & & & \\
\hline
\end{tabular}

UCLM: University of Castilla-La Mancha; UCA: University of Carthage; FIU: Florida International University; SD: Standard deviation; CI: Confidence interval; IR: Interquartile range; BMI: Body mass index. ${ }^{\dagger}$ Kruskal-Wallis test; ${ }^{\S} \chi^{2}$ test; $\$ \S$ Likelihood ratio test; * Significant differences. (1) - (5) Post hoc Dunn's test. (a) - (k) Data with the same superscript were significantly different. 
Table 4 shows daily total fiber intake distribution among the different categories for those variables presenting significant comparisons. Information on all the variables is shown in Tables S4-S6 in supplementary material. For each country, no statistically significant differences in fiber intake were found when smoking habits and level of physical activity variables were studied. Significant differences by gender were found in students from UCLM: male students showed higher daily total fiber intake than females. In Spain and Tunisia, no differences in fiber intake were found between students following and not following weight-loss diets. However, results were different among American participants: students not following weight-loss diets consumed less fiber than those who were (a difference of almost $7 \mathrm{~g} /$ day on average). Regardless of gender, a significantly positive association between energy intake and fiber intake was found in the three countries.

Table 4. Daily total fiber intake (g) by universities for variables presenting significant comparisons.

\begin{tabular}{|c|c|c|c|c|c|c|}
\hline \multicolumn{7}{|c|}{ UCLM (SPAIN) } \\
\hline & $n$ & Mean + SD & $95 \% \mathrm{CI}$ & Median & IR & $p$ \\
\hline \multicolumn{7}{|l|}{ Gender } \\
\hline Men & 120 & $16.4 \pm 6.3$ & $15.3-17.5$ & 14.7 & 8.4 & \multirow[t]{2}{*}{$0.047^{+, *}$} \\
\hline Women & 152 & $15.1 \pm 6.9$ & $14.0-16.2$ & 13.9 & 7.0 & \\
\hline \multicolumn{7}{|l|}{ Weight-loss diet } \\
\hline Yes & 14 & $15.3 \pm 6.6$ & $11.5-19.1$ & 14.4 & 10.3 & \multirow{2}{*}{$0.691^{+}$} \\
\hline No & 258 & $15.7 \pm 6.6$ & $14.9-16.5$ & 14.4 & 7.3 & \\
\hline \multicolumn{7}{|l|}{ BMI-categories } \\
\hline Underweight & 23 & $14.2 \pm 5.6$ & $11.8-16.7$ & 12.7 & 5.2 & \multirow{4}{*}{$\begin{array}{c}p \text {-trend } \\
0.051 \S\end{array}$} \\
\hline Normal range & 199 & $16.1 \pm 6.8$ & $15.1-17.0$ & 14.8 & 7.5 & \\
\hline Overweight & 40 & $14.8 \pm 6.3$ & $12.8-16.8$ & 13.2 & 8.3 & \\
\hline Obese & 10 & $14.5 \pm 5.8$ & $10.3-18.7$ & 12.1 & 8.7 & \\
\hline \multicolumn{7}{|l|}{ BMI-2-categories } \\
\hline Underw. + normal rg. & 222 & $15.9 \pm 6.7$ & $15.0-16.8$ & 14.5 & 7.2 & \multirow{2}{*}{$0.190^{+}$} \\
\hline Overweight + obese & 50 & $14.7 \pm 6.1$ & $13.0-16.5$ & 12.9 & 8.3 & \\
\hline BMI & \multicolumn{5}{|c|}{$r=-0.019$ (Spearman's correlation coefficient) } & 0.753 \\
\hline Energy intake & \multicolumn{5}{|c|}{$r=0.462$ (Spearman's correlation coefficient) } & $<0.001 * *$ \\
\hline \multicolumn{7}{|c|}{ UCA (TUNISIA) } \\
\hline & $n$ & Mean + SD & $95 \% \mathrm{CI}$ & Median & IR & $p$ \\
\hline \multicolumn{7}{|l|}{ Gender } \\
\hline Men & 46 & $14.6 \pm 5.6$ & $13.0-16.3$ & 14.0 & 5.7 & \multirow[t]{2}{*}{$0.352^{+}$} \\
\hline Women & 86 & $15.1 \pm 4.3$ & $14.2-16.1$ & 14.8 & 5.4 & \\
\hline \multicolumn{7}{|l|}{ Weight-loss diet } \\
\hline Yes & 16 & $14.6 \pm 6.2$ & $11.3-17.9$ & 14.7 & 7.1 & \multirow[t]{2}{*}{$0.734 \ddagger$} \\
\hline No & 116 & $15.0 \pm 4.6$ & $14.2-15.9$ & 14.5 & 5.3 & \\
\hline \multicolumn{7}{|l|}{ BMI-categories } \\
\hline Underweight & 21 & $15.3 \pm 4.7$ & $13.2-17.5$ & 13.6 & 6.4 & \multirow{4}{*}{$\begin{array}{c}p \text {-trend } \\
0.471^{\S}\end{array}$} \\
\hline Normal range & 99 & $15.0 \pm 4.6$ & $14.1-15.9$ & 14.9 & 5.3 & \\
\hline Overweight & 10 & $14.1 \pm 6.7$ & $9.3-18.9$ & 13.2 & 8.0 & \\
\hline Obese & 2 & $12.1 \pm 2.4$ & $0.0-33.7$ & 12.1 & - & \\
\hline \multicolumn{7}{|l|}{ BMI-2-categories } \\
\hline Underw. + normal rg. & 120 & $15.1 \pm 4.6$ & $14.3-15.9$ & 14.8 & 5.3 & \multirow[t]{2}{*}{$0.128^{+}$} \\
\hline Overweight + obese & 12 & $13.7 \pm 6.2$ & $9.8-17.7$ & 13.0 & 5.8 & \\
\hline BMI & \multicolumn{5}{|c|}{$r=-0.053$ (Spearman's correlation coefficient) } & 0.543 \\
\hline Energy intake & \multicolumn{5}{|c|}{$r=0.595$ (Spearman's correlation coefficient) } & $<0.001 * *$ \\
\hline \multicolumn{7}{|c|}{ FIU (USA) } \\
\hline & $n$ & Mean + SD & $95 \% \mathrm{CI}$ & Median & IR & $p$ \\
\hline \multicolumn{7}{|l|}{ Gender } \\
\hline Men & 73 & $20.2 \pm 11.6$ & $17.5-22.9$ & 17.3 & 14.8 & $0.393^{+}$ \\
\hline Women & 253 & $20.9 \pm 12.1$ & $19.4-22.4$ & 18.1 & 12.5 & \\
\hline Weight-loss diet & & & & & & \\
\hline Yes & 72 & $26.8 \pm 15.6$ & $23.2-30.5$ & 22.7 & 17.7 & $<0.001^{t, *}$ \\
\hline No & 254 & $19.0 \pm 10.2$ & $17.8-20.3$ & 16.7 & 11.3 & \\
\hline
\end{tabular}


Table 4. Cont

\begin{tabular}{|c|c|c|c|c|c|c|}
\hline \multicolumn{7}{|c|}{ FIU (USA) } \\
\hline & $n$ & Mean + SD & $95 \% \mathrm{CI}$ & Median & IR & $p$ \\
\hline \multicolumn{7}{|l|}{ BMI-categories } \\
\hline Underweight & 16 & $25.6 \pm 18.4$ & $15.7-35.4$ & 20.2 & 14.2 & \multirow{4}{*}{$\begin{array}{l}p \text {-trend } \\
0.322 \S\end{array}$} \\
\hline Normal range & 210 & $21.0 \pm 11.7$ & $19.4-22.6$ & 18.1 & 13.4 & \\
\hline Overweight & 66 & $19.6 \pm 12.7$ & $16.5-22.7$ & 15.3 & 13.0 & \\
\hline Obese & 34 & $19.3 \pm 8.5$ & $16.4-22.3$ & 18.3 & 8.7 & \\
\hline \multicolumn{7}{|l|}{ BMI-2-categories } \\
\hline Underw. + normal rg. & 226 & $21.3 \pm 12.3$ & $19.7-22.9$ & 18.5 & 13.3 & \multirow[t]{2}{*}{$0.087^{\dagger}$} \\
\hline Overweight + obese & 100 & $19.5 \pm 11.4$ & $17.2-21.8$ & 15.7 & 11.0 & \\
\hline BMI & \multicolumn{5}{|c|}{$r=-0.127$ (Spearman's correlation coefficient) } & $0.022 * *,(1)$ \\
\hline Energy intake & \multicolumn{5}{|c|}{$r=0.449$ (Spearman's correlation coefficient) } & $<0.001 * *$ \\
\hline
\end{tabular}

UCLM: University of Castilla-La Mancha; UCA: University of Carthage; FIU: Florida International University; SD: Standard deviation; CI: Confidence interval; IR: Interquartile range; BMI: Body mass index. ${ }^{\dagger}$ Mann-Whitney $\mathrm{U}$ test; ${ }^{\ddagger}$ Student's $t$ test; ${ }^{\S}$ Jonckheere-Terpstra trend test; ${ }^{*}$ Significant differences; ${ }^{* *}$ Significant correlation;

(1) Correlations by sex: Men: $r=0.098, p=0.408$; Women: $r=-0.178, p=0.004 * *$.

Although when the BMI variable was categorized, no significant differences in fiber intake was found among the four categories, a negative correlation between total fiber intake and BMI was found in students from FIU when BMI was considered as a continuous variable. Further analysis by gender showed a negative association between these variables in females but no association was found in males (Table 4).

Table 5 shows fiber intake $(\mathrm{g} / 1000 \mathrm{Kcal})$ by the food groups providing the largest amounts of fiber (cereals, legumes, vegetables and fruits) for all students from UCLM, FIU and UCA. In all cases, mean total fiber intake by country did not reach AI. A large percentage of Tunisian/Spanish (>96\%) and American $(>80 \%)$ subjects did not reach adequate fiber intake. Moreover, around $70 \%$ of Mediterranean students and more than $50 \%$ of American students did not even reach two thirds (9.3 g/1000 kcal) of AI (Figure 1).

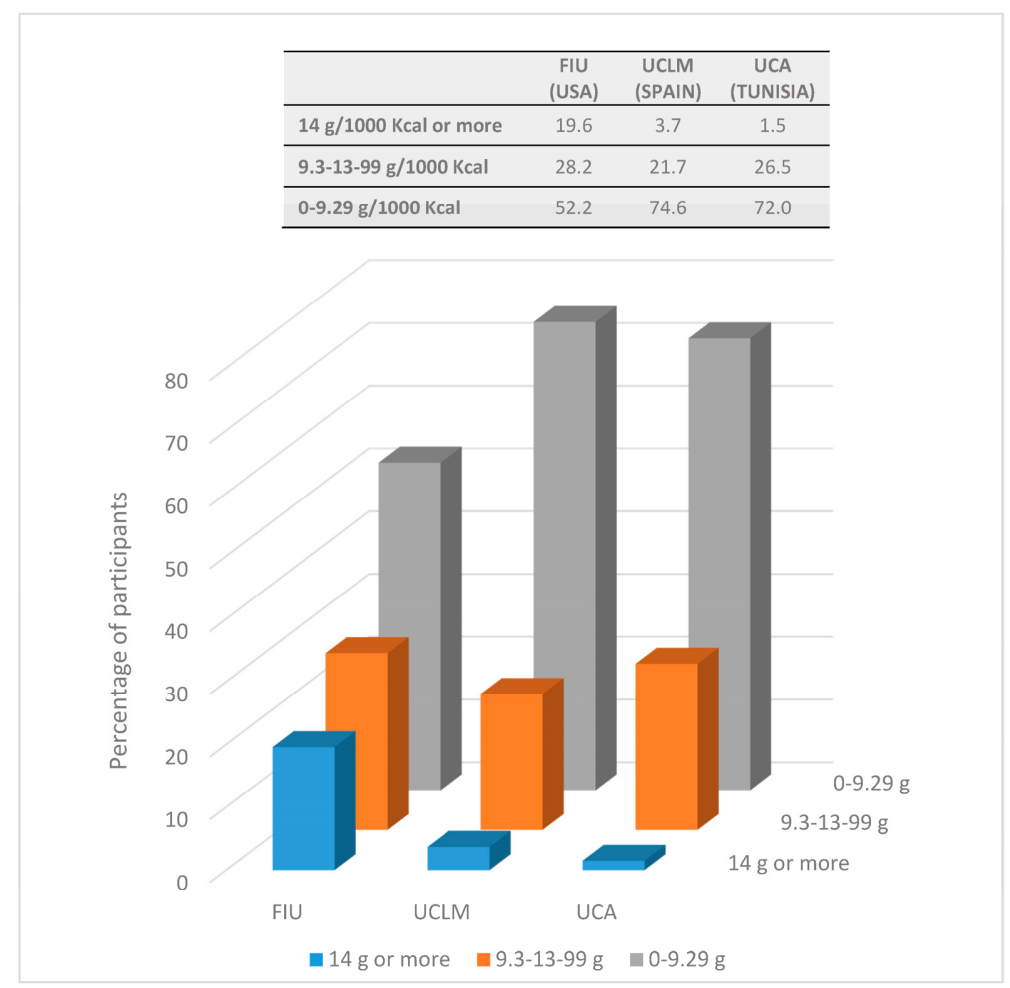

Figure 1. Percentage of participants consuming less than two thirds of the AI, those who consumed between two thirds of the AI and the AI, and those consuming at least the AI (14 g/1000 Kcal). AI: Adequate Intake; FIU: Florida International University; UCLM: University of Castilla-La Mancha; UCA: University of Carthage. 
Table 5. Fiber intakes $(\mathrm{g} / 1000 \mathrm{Kcal})$ from the main food groups providing fiber $\S$.

\begin{tabular}{|c|c|c|c|c|c|}
\hline & & UCLM (SPAIN) & UCA (TUNISIA) & FIU (USA) & $p^{\dagger}$ \\
\hline \multirow{5}{*}{ Total sample } & Cereals & $\begin{array}{c}3.3 \pm 1.3(3.1-3.4) \\
3.0(1.4)^{\mathrm{a}}\end{array}$ & $\begin{array}{c}3.6 \pm 1.1(3.4-3.8) \\
3.6(1.5)^{a}, b\end{array}$ & $\begin{array}{c}3.0 \pm 1.5(2.9-3.2) \\
2.9(2.1)^{\mathrm{b}}\end{array}$ & $p<0.001$ * \\
\hline & Legumes & $\begin{array}{c}0.5 \pm 1.1(0.4-0.6) \\
0.000(0.3)^{c}\end{array}$ & $\begin{array}{c}0.5 \pm 1.1(0.3-0.6) \\
0.000(0.4)\end{array}$ & $\begin{array}{l}1.2 \pm 2.5(0.9-1.4) \\
0.000(1.1)^{c}\end{array}$ & $p=0.001$ * \\
\hline & Vegetables & $\begin{array}{c}1.5 \pm 1.3(1.4-1.7) \\
1.2(1.6)\end{array}$ & $\begin{array}{c}1.5 \pm 1.0(1.4-1.7) \\
1.4(1.4)\end{array}$ & $\begin{array}{c}1.7 \pm 1.6(1.6-1.9) \\
1.3(1.7)\end{array}$ & $p=0.360$ \\
\hline & Fruits & $\begin{array}{c}1.1 \pm 1.4(0.9-1.3) \\
0.6(1.7)^{d}\end{array}$ & $\begin{array}{c}1.3 \pm 1.2(1.0-1.5) \\
1.0(1.6)^{\mathrm{e}}\end{array}$ & $\begin{array}{c}2.0 \pm 2.4(1.7-2.2) \\
1.3(2.2) \mathrm{d}, \mathrm{e}\end{array}$ & $p<0.001$ * \\
\hline & Total intake & $\begin{array}{c}8.1 \pm 3.2(7.7-8.5) \\
7.5(3.4)^{\mathrm{f}}\end{array}$ & $\begin{array}{c}8.4 \pm 2.2(8.0-8.7) \\
8.0(2.7)^{\mathrm{g}}\end{array}$ & $\begin{array}{c}10.5 \pm 5.7(9.9-11.1) \\
8.9(5.9)^{\mathrm{f}, \mathrm{g}}\end{array}$ & $p<0.001 *$ \\
\hline \multirow{5}{*}{$0-9.29 \mathrm{~g}$} & Cereals & $\begin{array}{c}3.2 \pm 1.1(3.0-3.3) \\
3.0(1.4)^{\mathrm{h}}\end{array}$ & $\begin{array}{c}3.5 \pm 1.1(3.3-3.7) \\
3.5(1.5)^{h, i}\end{array}$ & $\begin{array}{c}3.0 \pm 1.3(2.8-3.1) \\
2.9(1.8)^{\mathrm{i}}\end{array}$ & $p<0.001$ * \\
\hline & Legumes & $\begin{array}{c}0.2 \pm 0.7(0.2-0.3) \\
0.000(0.00)\end{array}$ & $\begin{array}{c}0.2 \pm 0.4(0.1-0.3) \\
0.000(0.00)\end{array}$ & $\begin{array}{c}0.2 \pm 0.5(0.2-0.3) \\
0.000(0.04)\end{array}$ & $p=0.224$ \\
\hline & Vegetables & $\begin{array}{c}1.2 \pm 1.0(1.1-1.4) \\
1.1(1.3)\end{array}$ & $\begin{array}{c}1.3 \pm 0.8(1.1-1.5) \\
1.2(1.3)\end{array}$ & $\begin{array}{c}1.2 \pm 0.9(1.1-1.4) \\
1.1(1.2)\end{array}$ & $p=0.670$ \\
\hline & Fruits & $\begin{array}{c}0.8 \pm 1.0(0.6-0.9) \\
0.5(1.1)^{j, k}\end{array}$ & $\begin{array}{c}1.1 \pm 1.0(0.9-1.3) \\
0.9(1.4)^{j}\end{array}$ & $\begin{array}{c}0.9 \pm 0.9(0.8-1.1) \\
0.7(1.3)^{\mathrm{k}}\end{array}$ & $p=0.004$ * \\
\hline & Total intake & $\begin{array}{c}6.7 \pm 1.5(6.5-6.9) \\
6.7(2.4)^{1}\end{array}$ & $\begin{array}{c}7.3 \pm 1.3(7.0-7.5) \\
7.4(1.9)^{\mathrm{l}, \mathrm{m}}\end{array}$ & $\begin{array}{c}6.7 \pm 1.5(6.5-6.9) \\
6.8(2.1)^{\mathrm{m}}\end{array}$ & $p=0.003$ * \\
\hline \multirow{5}{*}{$9.30-13.99 \mathrm{~g}$} & Cereals & $\begin{array}{c}3.7 \pm 1.8(3.3-4.2) \\
3.4(1.7)\end{array}$ & $\begin{array}{c}3.9 \pm 1.1(3.5-4.3) \\
3.9(1.7)^{n}\end{array}$ & $\begin{array}{c}3.1 \pm 1.6(2.8-3.4) \\
3.0(2.3)^{n}\end{array}$ & $p=0.007$ * \\
\hline & Legumes & $\begin{array}{c}1.0 \pm 1.4(0.6-1.3) \\
0.5(1.7)\end{array}$ & $\begin{array}{c}1.2 \pm 1.8(0.6-1.8) \\
0.5(1.5)\end{array}$ & $\begin{array}{c}1.2 \pm 1.8(0.8-1.5) \\
0.000(1.6)\end{array}$ & $p=0.867$ \\
\hline & Vegetables & $\begin{array}{c}2.3 \pm 1.7(1.9-2.7) \\
2.0(1.4)\end{array}$ & $\begin{array}{c}2.2 \pm 1.1(1.8-2.6) \\
2.1(1.9)\end{array}$ & $\begin{array}{c}1.9 \pm 1.5(1.6-2.2) \\
1.5(1.9)\end{array}$ & $p=0.057$ \\
\hline & Fruits & $\begin{array}{c}2.0 \pm 1.8(1.5-2.4) \\
2.0(2.7)\end{array}$ & $\begin{array}{c}1.6 \pm 1.4(1.1-2.1) \\
1.3(2.6)^{\circ}\end{array}$ & $\begin{array}{c}2.5 \pm 1.9(2.1-2.9) \\
2.3(2.9)^{\circ}\end{array}$ & $p=0.027^{*}$ \\
\hline & Total intake & $\begin{array}{c}11.1 \pm 1.3(10.8-11.4) \\
10.9(2.2)\end{array}$ & $\begin{array}{c}10.9 \pm 1.3(10.5-11.4) \\
10.5(2.1)\end{array}$ & $\begin{array}{c}11.4 \pm 1.2(11.1-11.6) \\
11.3(2.0)\end{array}$ & $p=0.134$ \\
\hline \multirow{5}{*}{$\geq 14 \mathrm{~g}$} & Cereals & $\begin{array}{c}3.5 \pm 1.8(2.2-4.8) \\
3.5(2.7)\end{array}$ & $\begin{array}{c}4.2 \pm 0.2(2.6-5.7) \\
4.2(-)\end{array}$ & $\begin{array}{c}3.1 \pm 2.1(2.6-3.6) \\
3.1(2.6)\end{array}$ & $p=0.366$ \\
\hline & Legumes & $\begin{array}{c}2.2 \pm 2.7(0.3-4.1) \\
1.2(4.4)\end{array}$ & $\begin{array}{c}0.2 \pm 0.3(-2.5-3.0) \\
0.2(-)\end{array}$ & $\begin{array}{c}3.7 \pm 4.2(2.6-4.7) \\
2.4(6.5)\end{array}$ & $p=0.426$ \\
\hline & Vegetables & $\begin{array}{c}1.9 \pm 1.5(0.9-3.0) \\
1.5(1.9)\end{array}$ & $\begin{array}{c}2.7 \pm 0.6(-3.03-8.5) \\
2.7(-)\end{array}$ & $\begin{array}{c}2.9 \pm 2.2(2.3-3.4) \\
2.5(2.8)\end{array}$ & $p=0.378$ \\
\hline & Fruits & $\begin{array}{c}3.0 \pm 2.4(1.3-4.8) \\
2.8(4.2)\end{array}$ & $\begin{array}{c}5.0 \pm 1.0(-3.6-13.6) \\
5.0(-)\end{array}$ & $\begin{array}{c}4.1 \pm 3.8(3.1-5.0) \\
3.3(4.0)\end{array}$ & $p=0.474$ \\
\hline & Total intake & $\begin{array}{c}18.2 \pm 6.1(13.8-22.5) \\
15.5(4.4)\end{array}$ & $\begin{array}{c}14.7 \pm 0.8(7.3-22.2) \\
14.7(-)\end{array}$ & $\begin{array}{c}19.4 \pm 5.6(18.0-20.8) \\
18.0(5.6)\end{array}$ & $p=0.093$ \\
\hline
\end{tabular}

UCLM: University of Castilla-La Mancha; UCA: University of Carthage; FIU: Florida International University.

${ }^{+}$Kruskal-Wallis and post hoc Dunn. Data with the same superscript were significantly different; ${ }^{*}$ Significant differences; $\S$ All values are mean \pm standard deviation (95\% confidence interval) and median (interquartile range).

The study of the individuals not reaching two thirds of AI showed significant differences between Tunisian and Spanish/American students: subjects from UCA consumed about $0.6 \mathrm{~g} / 1000 \mathrm{Kcal}$ more than the students from UCLM or FIU (Table 5).

Regarding the origin of the fiber, our results showed that $76.6 \%$ of the fiber came from cereals, legumes, vegetables and fruits (including nuts). This percentage reached $82.6 \%$ in students from UCA. In all countries, cereals provided an average of at least $3 \mathrm{~g} / 1000 \mathrm{Kcal}$. The sum of vegetables and fruits provided $3.7 \mathrm{~g} / 1000 \mathrm{Kcal}$ of fiber in students from FIU and 2.8/2.6 g/1000 Kcal in Tunisian/Spanish participants, respectively. It is interesting to note that fiber consumption from legumes reported by American students was more than twice that reported by Mediterranean students. In the three countries, cereals were the main source of fiber for people with fiber intake below AI. Regarding individuals reaching AI, cereals were the major source of fiber among Spanish students while the main source of fiber among American and Tunisian students was fruit.

Consumption of fiber $(\mathrm{g})$ from the main groups that contribute to total fiber intake is shown in Figure 2. The distribution of the consumption of high-fiber food groups by quartiles of total fiber intake is shown in Table 6 . For each country, total fiber intake was significantly different when quartiles were 
compared. However, participants from different countries showed some differences in the main food groups that contributed to increase total fiber intake. Given that recent investigations have shown that nuts play an important role in health [68], Table 6 and Figure 2 show specific additional information about fiber intake from nuts.

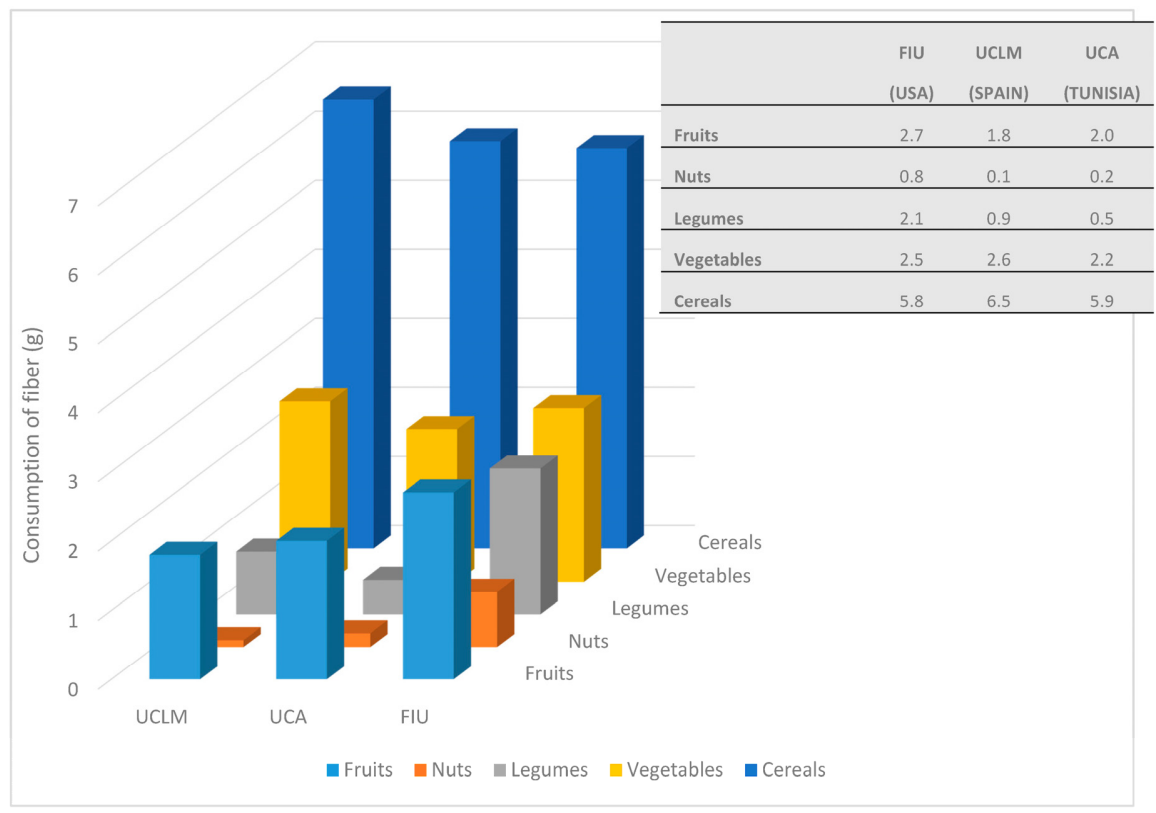

Figure 2. Consumption of fiber $(\mathrm{g})$ from the main food groups that contribute to total fiber intake.

Table 6. Distribution of consumption of high-fiber groups by quartiles of total fiber intake $(\mathrm{g}) \S$.

\begin{tabular}{|c|c|c|c|c|c|c|}
\hline \multicolumn{7}{|c|}{ UCLM (SPAIN) } \\
\hline \multirow{3}{*}{ Total } & TOTAL & Q1 & Q2 & Q3 & Q4 & $p$-trend ${ }^{\dagger}$ \\
\hline & $15.7 \pm 6.6(14.9-16.5)$ & $9.1 \pm 1.6(8.8-9.5)$ & $12.8 \pm 0.9(12.6-13.0)$ & $16.5 \pm 1.4(16.1-16.8)$ & $24.3 \pm 6.7(22.6-25.9)$ & \multirow{2}{*}{$<0.001$ * } \\
\hline & $14.4(7.4)$ & $9.5(2.2)$ & $12.9(1.4)$ & $16.4(2.0)$ & $22.7(6.9)$ & \\
\hline \multirow{2}{*}{ Fruits } & $1.8 \pm 2.4(1.6-2.1)$ & $0.8 \pm 1.1(0.6-1.1)$ & $1.4 \pm 1.8(0.9-1.8)$ & $1.8 \pm 2.2(1.2-2.3)$ & $3.4 \pm 3.2(2.6-4.2)$ & \multirow{2}{*}{$<0.001$ * } \\
\hline & $0.9(2.9)$ & $0.2(1.4)$ & $0.8(2.1)$ & $0.7(3.6)$ & $2.5(5.0)$ & \\
\hline \multirow{2}{*}{ Nuts } & $0.1 \pm 0.4(0.1-0.2)$ & $0.1 \pm 0.4(0.0-0.2)$ & $0.2 \pm 0.6(0.0-0.3)$ & $0.1 \pm 0.3(0.0-0.2)$ & $0.1 \pm 0.3(0.0-0.2)$ & \multirow{2}{*}{0.168} \\
\hline & $0.0(0.0)$ & $0.0(0.0)$ & $0.0(0.0)$ & $0.0(0.0)$ & $0.0(0.0)$ & \\
\hline \multirow{2}{*}{ Legumes } & $0.9 \pm 2.2(0.7-1.2)$ & $0.2 \pm 0.7(0.0-0.3)$ & $0.2 \pm 0.8(0.04-0.4)$ & $0.7 \pm 1.4(0.4-1.1)$ & $2.6 \pm 3.5(1.8-3.5)$ & \multirow{2}{*}{$<0.001$ * } \\
\hline & $0.0(0.8)$ & $0.0(0.0)$ & $0.0(0.0)$ & $0.0(0.0)$ & $1.6(3.9)$ & \\
\hline \multirow{2}{*}{ Vegetables } & $2.6 \pm 1.9(2.4-2.8)$ & $1.7 \pm 1.4(1.4-2.1)$ & $2.4 \pm 1.9(2.0-2.9)$ & $3.0 \pm 2.0(2.5-3.5)$ & $3.1 \pm 2.0(2.6-3.6)$ & \multirow{2}{*}{$<0.001$ * } \\
\hline & $2.2(2.6)$ & $1.4(2.2)$ & $2.0(2.0)$ & $2.5(3.1)$ & $2.6(2.9)$ & \\
\hline \multirow{2}{*}{ Cereals } & $6.5 \pm 3.2(6.1-6.9)$ & $4.6 \pm 1.7(4.2-5.0)$ & $6.1 \pm 2.5(5.5-6.7)$ & $7.0 \pm 2.9(6.3-7.7)$ & $8.5 \pm 4.0(7.5-9.4)$ & \multirow{2}{*}{$<0.001$ * } \\
\hline & $6.0(3.9)$ & $4.6(2.1)$ & $6.0(3.4)$ & $6.4(4.7)$ & $8.1(5.7)$ & \\
\hline \multicolumn{7}{|c|}{ UCA (TUNISIA) } \\
\hline \multirow{3}{*}{ Total } & TOTAL & Q1 & Q2 & Q3 & Q4 & $p$-trend ${ }^{\dagger}$ \\
\hline & $15.0 \pm 4.8(14.1-15.8)$ & $9.5 \pm 1.8(8.9-10.2)$ & $13.3 \pm 0.8(13.0-13.5)$ & $15.9 \pm 0.9(15.6-16.2)$ & $21.1 \pm 3.8(19.8-22.5)$ & \multirow{2}{*}{$<0.001 *$} \\
\hline & $14.5(5.4)$ & $9.5(2.3)$ & $13.5(1.5)$ & $15.7(1.8)$ & $20.1(4.6)$ & \\
\hline \multirow{2}{*}{ Fruits } & $2.0 \pm 2.1(1.7-2.4)$ & $0.7 \pm 1.1(0.3-1.1)$ & $1.9 \pm 1.6(1.3-2.4)$ & $2.1 \pm 1.9(1.4-2.8)$ & $3.5 \pm 2.5(2.6-4.4)$ & \multirow{2}{*}{$<0.001$ * } \\
\hline & $1.6(3.0)$ & $0.000(1.5)$ & $1.6(2.7)$ & $1.7(2.4)$ & $2.8(2.9)$ & \\
\hline \multirow{2}{*}{ Nuts } & $0.2 \pm 0.8(0.0-0.3)$ & $0.04 \pm 0.2(0.0-0.1)$ & $0.2 \pm 0.6(0.0-0.4)$ & $0.1 \pm 0.4(0.0-0.2)$ & $0.5 \pm 1.5(0.0-1.0)$ & \multirow{2}{*}{0.351} \\
\hline & $0.0(0.0)$ & $0.0(0.0)$ & $0.0(0.0)$ & $0.0(0.0)$ & $0.0(0.0)$ & \\
\hline \multirow{2}{*}{ Legumes } & $0.5 \pm 1.1(0.3-0.7)$ & $0.1 \pm 0.5(0.0-0.3)$ & $0.6 \pm 1.0(0.2-0.9)$ & $0.3 \pm 0.7(0.1-0.6)$ & $1.0 \pm 1.7(0.4-1.6)$ & \multirow{2}{*}{$0.007^{*}$} \\
\hline & $0.0(0.7)$ & $0.0(0.0)$ & $0.0(0.7)$ & $0.0(0.3)$ & $0.0(1.6)$ & \\
\hline \multirow{2}{*}{ Vegetables } & $2.2 \pm 1.5(20 .-2.5)$ & $1.5 \pm 1.1(1.1-1.8)$ & $2.0 \pm 1.3(1.6-2.5)$ & $2.2 \pm 1.3(1.7-2.7)$ & $3.3 \pm 1.6(2.7-3.9)$ & \multirow{2}{*}{$<0.001$ * } \\
\hline & $1.9(2.2)$ & $1.2(1.2)$ & $1.9(2.0)$ & $1.8(2.0)$ & $3.2(2.5)$ & \\
\hline \multirow{2}{*}{ Cereals } & $5.9 \pm 2.2(5.5-6.3)$ & $4.9 \pm 2.0(4.2-5.6)$ & $5.2 \pm 1.9(4.6-5.9)$ & $6.8 \pm 2.3(5.9-7.6)$ & $6.8 \pm 2.0(6.1-7.5)$ & \multirow{2}{*}{$<0.001$ * } \\
\hline & $6.0(3.0)$ & $4.6(3.4)$ & $5.4(3.0)$ & $6.0(3.2)$ & $6.8(2.8)$ & \\
\hline
\end{tabular}


Table 6. Cont.

\begin{tabular}{|c|c|c|c|c|c|c|}
\hline \multicolumn{7}{|c|}{ FIU (USA) } \\
\hline \multirow{2}{*}{ Total } & TOTAL & Q1 & Q2 & Q3 & Q4 & $p$-trend ${ }^{\dagger}$ \\
\hline & $20.8 \pm 12.0(19.5-22.1)$ & $10.0 \pm 2.2(9.5-10.5)$ & $15.3 \pm 1.5(15.0-15.6)$ & $21.1 \pm 2.3(20.6-21.6)$ & $36.8 \pm 12.8(34.0-39.6)$ & $<0.001 *$ \\
\hline \multirow{2}{*}{ Fruits } & $2.7 \pm 3.0(2.4-3.0)$ & $1.0 \pm 1.2(0.7-1.3)$ & $2.1 \pm 1.8(1.7-2.5)$ & $3.4 \pm 3.0(2.7-4.0)$ & $4.3 \pm 4.0(3.4-5.2)$ & \multirow{2}{*}{$<0.001$ * } \\
\hline & $1.8(3.0)$ & $0.7(1.6)$ & $1.6(2.2)$ & $2.5(3.2)$ & $3.6(4.8)$ & \\
\hline Nuts & $0.8 \pm 1.4(0.3-0.6)$ & $0.05 \pm 0.3(0-0.1)$ & $0.3 \pm 0.9(0.1-0.5)$ & $0.6 \pm 1.6(0.2-0.9)$ & $1.0 \pm 2.0(0.5-1.4)$ & $<0.001 *$ \\
\hline \multirow{2}{*}{ Legumes } & $2.1 \pm 4.6(1.6-2.6)$ & $0.3 \pm 0.7(0.2-0.5)$ & $0.6 \pm 1.0(0.3-0.8)$ & $1.3 \pm 2.6(0.7-1.9)$ & $6.3 \pm 7.3(4.7-7.9)$ & \multirow{2}{*}{$<0.001$ * } \\
\hline & $0.000(1.8)$ & $0.0(0.0)$ & $0.0(1.1)$ & $0.0(1.8)$ & $3.8(12.4)$ & \\
\hline \multirow{2}{*}{ Vegetables } & $2.5 \pm 2.1(2.3-2.8)$ & $1.7 \pm 1.4(1.4-2.00)$ & $2.6 \pm 2.1(2.1-3.1)$ & $2.9 \pm 1.9(2.4-3.3)$ & $3.1 \pm 2.6(2.5-3.6)$ & \multirow{2}{*}{$<0.001 *$} \\
\hline & $2.0(2.7)$ & $1.5(1.5)$ & $2.1(2.8)$ & $2.7(3.1)$ & $2.8(3.3)$ & \\
\hline Cereals & $5.8 \pm 3.1(5.5-6.2)$ & $4.6 \pm 2.0(4.2-5.1)$ & $5.6 \pm 2.9(4.9-6.2)$ & $6.8 \pm 3.0(6.1-7.4)$ & $6.4 \pm 3.7(5.6-7.2)$ & $<0.001 *$ \\
\hline
\end{tabular}

UCLM: University of Castilla-La Mancha; UCA: University of Carthage; FIU: Florida International University; ${ }^{\dagger}$ Jonckheere-Terpstra trend test; * Significant differences; ${ }^{\S}$ All values are mean \pm standard deviation $(95 \%$ confidence interval) and median (interquartile range).

In line with previous results, when fiber intake per thousand kilocalories was studied (Table 5), the results in Table 6 also show that in our population the main source of total fiber was cereals, followed by vegetables and fruits. For each country (Spain, Tunisia and the USA), the amount of total fiber obtained from cereals $(6.5,5.9$ and $5.8 \mathrm{~g}$ on average, respectively) was two to three times higher than the amount of fiber from the groups that ranked second: vegetables $(2.6,2.2$ and $2.5 \mathrm{~g}$ on average, respectively) and fruits (1.8, 2.0 and $2.7 \mathrm{~g}$ on average, respectively).

It is interesting to note that, in students from UCA, the increased consumption of fiber from the first to the fourth quartiles came from fruit consumption (fiber intake increases $2.8 \mathrm{~g}$ on average), followed by vegetables and cereals (fiber intake increases around $2 \mathrm{~g}$ on average in each group). In relative terms, fiber obtained from fruits increased five-fold between the first and the last quartiles while fiber from vegetables and cereals increased 2.2 and 1.4 times, respectively. In relative terms, fiber obtained from legumes and nuts increased ten-fold between the first and the last quartiles. These intakes increased by nearly $1 \mathrm{~g}$ and $0.5 \mathrm{~g}$, respectively, in absolute terms.

In students from FIU, more food groups contributed to the increase in total fiber from the first to the fourth quartiles. In the legume group, mean fiber intake in the first quartile was 0.3 , while mean fiber intake in the last quartile was 6.3, which is an increase of $6 \mathrm{~g}$ (in relative terms, this fiber from legumes increased around 20 times). Mean fiber consumption from fruits increased from $1.0 \mathrm{~g}$ to $4.3 \mathrm{~g}$, mean fiber consumption from vegetables increased from $1.7 \mathrm{~g}$ to $3.1 \mathrm{~g}$, and mean fiber consumption from cereals increased from $4.6 \mathrm{~g}$ to $6.4 \mathrm{~g}$. In relative terms, fiber from these groups increased 4.3 , 1.8 and 1.4 times, respectively. Finally, in relative terms, fiber obtained from nuts increased 21-fold between the first and the last quartiles. This intake was an increase of nearly $1 \mathrm{~g}$ in absolute terms.

Among participants from UCLM, the increased consumption of fiber from the first to the fourth quartiles came from cereal consumption (in absolute terms, an increase of almost $4 \mathrm{~g}$ between the first and the fourth quartiles was found), followed by fruits, legumes and vegetables (differences of 2.6, 2.4 and $1.4 \mathrm{~g}$, respectively, were observed). In relative terms, in line with the results for American participants, the most important increase was found in the legume group (fiber obtained from legumes increased 13-fold between the first and the last quartiles), followed by the fruit group (fiber from fruits increased 4.3 times) and vegetable and cereal groups (fiber from these groups increased 1.8 times). It is interesting to highlight that fiber from nuts remained practically constant in all quartiles.

Although cereals, legumes, vegetables and fruit are the main fiber sources, young people usually consume pre-cooked foodstuff or ready-to-eat meals. Figure 3 shows fiber intake from appetizers, ready-to-eat meals, and sauces and condiments. The last group includes both healthy aromatic herbs, spices and seasonings, and complex sauces. In supplementary material Table S7 shows detailed information about these groups. Our results showed that even though intake for the main fiber food 
groups was highly homogenous across participants, differences appear when these other groups are studied. A total of $16.7 \%$ of fiber consumed by American participants was obtained from appetizers, prepared and precooked meals, and sauces and spices and condiments groups. This percentage was relatively small in Mediterranean participants. Spanish participants only reached $7.4 \%$ and Tunisian participants barely reached $2.6 \%$. It is worth highlighting the virtually non-existent fiber intake from both prepared and precooked meals and sauces among Tunisian participants. If we omit the spices and condiments group, fiber intake from prepared and precooked products by students from FIU is almost 25 times higher than among students from UCA and double that of students from UCLM.

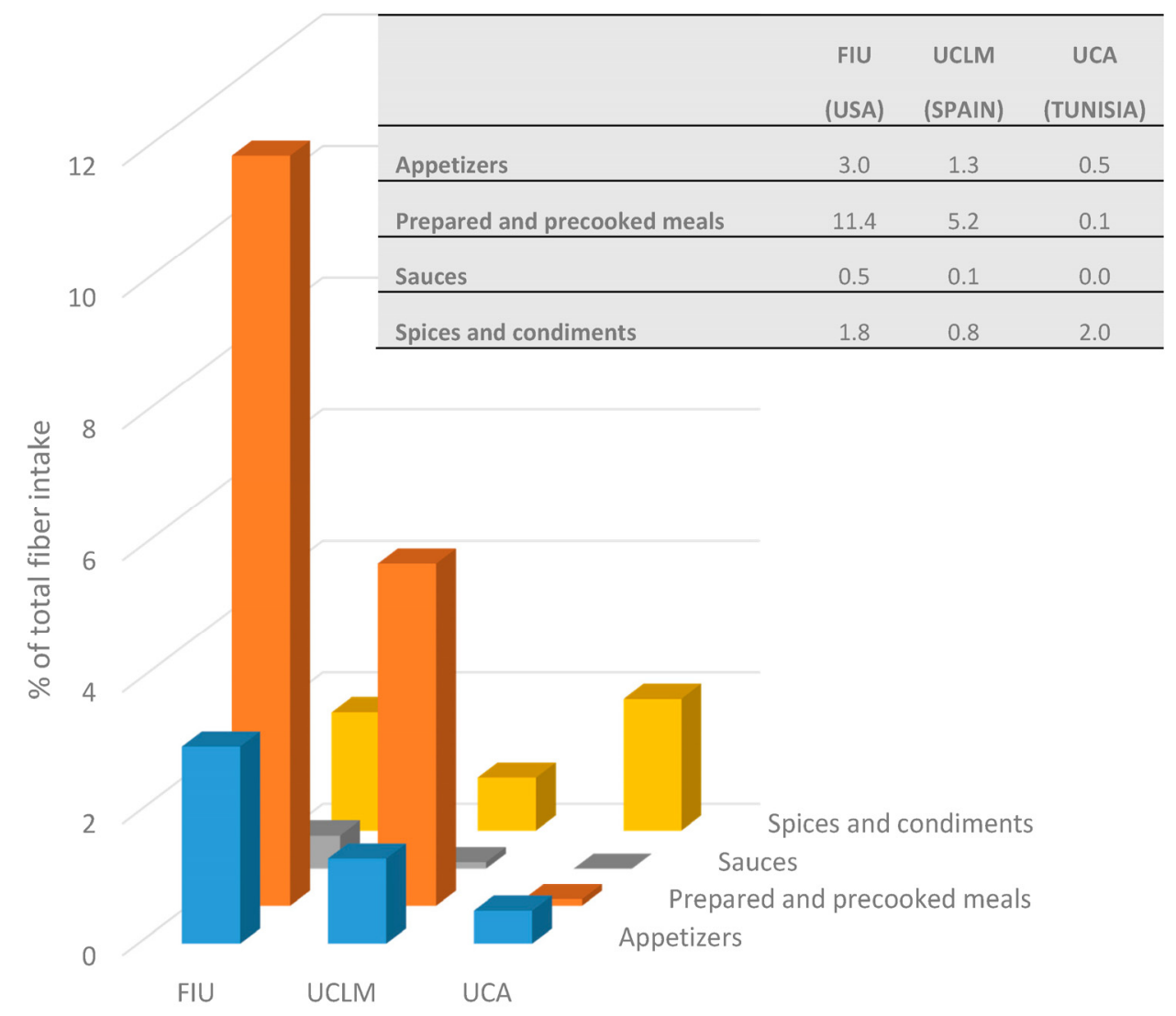

Figure 3. Fiber from appetizers, prepared and precooked meals, sauces, and spices and condiments (\% of total fiber intake).

\section{Discussion}

Economic and industrial development has led to changes in demographic structures, health and food patterns. New models of transitional nutrition characterized by loss of food culture are emerging. Decreased fiber intake is one of the characteristics of these new models traditionally linked to new health risks $[69,70]$. Benefits of dietary fiber intake go beyond effects on chronic diseases associated with developed economies (type 2 diabetes, cardiovascular diseases, cancer and others) $[9,10]$, besides the well-known impact on gastrointestinal diseases [1,2,25]. Recent research also suggests positive effects on other illnesses and conditions (infections or respiratory diseases) and negative effects on all the total death rates [12,71]. In this context, the pattern of fiber intake is considered an indicator of diet quality $[16,27]$ and international policy guidelines $[14,15]$ have included the intake of food sources with high fiber content in order to meet the recommendations.

Although qualitative differences between fiber food sources have been found according to availability, cultural and social characteristics, taboos and the economic development of countries, young adults are highly sensitive to trends, ready-to-eat meals being an example. Factors such as 
leaving school, going to university, leaving the parental home and having no cooking skills lead young people to be more affected by an unhealthy food environment $[13,41]$. The fiber pattern intake will persist for a lifetime in many cases [72]. Currently, there is great scientific interest in research on fiber and its food sources, as some long-term benefits of the former may depend on the latter [73,74]. Therefore, the pattern of fiber intake in young adults, like other nutrients, plays a key role in improving future health state.

The present study on diet quality measured by fiber patterns in young adults was carried out with data from students enrolled at the University of Castilla-La Mancha (Spain), the University of Carthage (Tunisia) and Florida International University (USA). There is extensive research on food patterns in university students around the world. Some works record fiber intake, in addition to other nutritive diet features [38-40,42,75], while others study the effect of fiber intake on certain diseases $[12,76,77]$ or investigate food sources of fiber in a specific country $[33,56,57,78]$. However, to the best of our knowledge, none of them has specifically addressed fiber consumption patterns in young adults with a high level of education but with different cultures and incomes, living in three countries with different levels of development and located on three continents. Tunisia is a developing country currently classified as low-middle income (but classified as middle-income when data were collected) [48], where traditional Mediterranean culture is supplemented by some French influences from recent centuries. Spain is another Mediterranean country, classified as high-income, and the USA is considered the prototype of the Western food model. Specific issues characterize the region where each University is located. The UCLM is located in a Spanish area in economic transition within the European Union [79]. A high percentage of students come from the same region. Miami, where FIU is situated, is inhabited by a large proportion of Latin Americans together other ethnic and cultural communities. UCA is located in the capital of Tunisia, one of the largest urban areas in the country [80].

The relationship between economic development and type of food consumed, with different consequences for health, generates models of transitional nutrition characterized by loss of both cultural and healthy food habits [69]. The impact in middle- and high-income areas leads to the adoption of unhealthier lifestyles and a shift away from healthier traditional ones. Thus, transition from the Mediterranean food pattern to the Western model implies a reduction in fiber intake and increased overall intake of saturated fat and sugar from precooked meals, snacks and other ready-toeat meals. Regarding the age range of the population studied, young adults are the most susceptible to advertising, cultural trends and attitudes, so rapid changes in food behavior towards new tendencies are common [37,81]. Moreover, the positive relationship between level of education and healthy food habits is also well established [82]. The large number of potential sources of interpersonal variation that emerge when analyzing a population homogenous in aspects such as level of education and age, but different in cultural features and economic development [58], may provide an excellent opportunity to deepen knowledge of the complex relationship between health and diet. Our work addresses the study of patterns of fiber intake in young adults through analysis of their food sources, and discusses the impact of culture and economic development of the countries and other factors on these patterns.

According to their pattern of fiber intake, the diet quality of the population studied is low, since the participants did not reach the AI, in line with other populations of similar characteristics $[42,57,75]$. However, cultural and economic factors affect patterns of fiber intake. Contrary to expectations, students from FIU consumed more fiber than students from UCA and UCLM, countries that share Mediterranean traditional culture and have lower incomes than USA. Our results also revealed that total fiber intake increased with energy intake but did not depend on smoking habits and physical activity in any country. Moreover, only among the USA participants did the following of a weight-loss diet influence the pattern of fiber intake.

Regardless of country, the main food groups contributing fiber to the diet were cereals, legumes, vegetables and fruits. The distribution of the main sources of fiber consumed by our students was different in each country. The cereal group provided the largest amount of fiber in the three countries, 
but while vegetables were the second group providing fiber among Spanish and Tunisian students, the fruit group was the second group among American students.

Generally, analyses conducted at the levels of nutrients, food groups and possible overall dietary patterns yield the most complete information about diet [58]. Therefore, these three levels of study have been detailed in the following discussion. In the first level, fiber as a nutrient has been considered and data about fiber intake have been analyzed. The second level comprises the study of food groups providing fiber and, in the third level, the analysis of dietary patterns is addressed.

\subsection{First Level}

Our students showed low levels of fiber intake. Only one out of ten participants consumed an adequate amount of fiber, as defined by recommendations. The mean intake of participants did not reach the recommended levels (AI higher or equal to $14 \mathrm{~g} / 1000 \mathrm{Kcal}$ ), which are based on scientific evidence of beneficial effects of fiber on chronic degenerative diseases [20]. At first sight, fiber intake runs parallel to the trend towards lower consumption of fiber associated with economic development of countries [81]. Thus, nutritional deficiencies associated with poverty are resolved, but, simultaneously, this environment involves harmful effects on health. Taking into account the level of education [82] , our results are in line with data on mean fiber intake found in other studies performed with young adults in these countries $[56,57,75,83]$ and also with university students from other European countries such as Poland [42] and Greece [84]. However, in our survey total fiber intake was lower than levels of consumption reported in other European countries among adult population [85].

The results for mean fiber intake found in each of the three countries studied were significantly different and, contrary to expectations, showed that American participants were the largest consumers of fiber. The study of the range of variation between the students with the lowest and the highest levels of fiber intake showed different results in UCA when compared with UCLM and FIU. In this sense, Tunisian participants showed a lower range of variation than Spanish and American participants, with the Americans exhibiting the widest range of variation. Differences in income among the countries could be one of the influencing factors.

Our study demonstrates the impact of a country's economic development on BMI, since one third of the American participants recorded excess weight, compared with one of ten of the Tunisians, with the Spanish students occupying the intermediate position. Only 5\% of American participants followed weight-loss diets. In American students fiber consumption also decreased when body weight increased and only overweight and obese females showed a negative association between fiber intake and BMI. This gender-related difference coincides with other previous studies in young and middle-aged US adults [35], where a low-fiber and high-fat diet was associated with the greatest risk of excess weight in women. Studies among obese Tunisian females reveal similar results [86]. This association was not found in Spanish, Tunisian, and non-overweight American participants, which is in line with results from other surveys conducted in Japanese or Swedish populations [77,87]. In young and adult populations from Northern European countries (Germany, Denmark and the UK), a negative association between fiber intake and weight has also been found, but other studies performed in Mediterranean countries such as Italy reported no association between weight and fiber intake [85]. These differences could be explained, at least in part, by the low proportion of people classified as overweight or obese among our Mediterranean participants, as also happens among Italian participants [85] and Swedish and Japanese populations [77,87].

A negative correlation between BMI and fiber intake was found in each country. However, USA students showed the highest mean BMI and the highest mean fiber intake, in spite of the Western-diet consumption. Most probably, differences in food sources derived from the economic development may account for the paradoxical clue.

As is often the case with most nutrients in free-living populations, fiber intake increased with energy intake in our students, independently of country and gender, although other studies have found a negative correlation between fiber intake and energy intake in females [88]. 


\subsection{Second Level}

Incomplete understanding of the effects of healthy fiber has guided research towards the investigation of food sources of fiber. In this section, we have discussed the main food groups contributing to total dietary fiber intake (cereals, legumes, vegetables and fruit). In addition, other minority groups were studied (appetizers, ready-to-eat meals, and sauces and condiments), due to possible different effects on health resulting from their consumption [36,37,89]. Regarding the main food groups, and according to current knowledge, different health benefits of fiber depend on criteria of viscosity and fermentability. Less fermentable fibers increase fecal weight to a greater degree than more fermentable fibers. Thus, the effect on fecal weight of fiber from cereals and vegetables is similar, and is higher than the effect of fiber from fruits [32]. Moreover, high consumption of fruits or fiber-fruit has been found to decrease the risk of cardiovascular disease in young Mediterranean people [33]. In our study, regardless of the country, the cereal group provided the highest amount of consumed fiber. It is interesting to highlight the high levels of fiber from legumes (string beans) consumed by our American students, which is in line with other studies in child and adult populations [16]. These outcomes matched those found in different surveys in the same countries and carried out among young and/or adult population when main food groups were studied $[12,57,75]$. However, when we considered the individuals reaching the recommended fiber intake, Tunisian participants showed the highest levels of fiber intake from fruits. This might provide greater protection against cardiovascular diseases in these young Mediterranean students.

Controversy in some results and the incidence of related chronic diseases has led researchers to suggest that quantity of fiber and the effect of its chemical properties on health are insufficient to fully understand its healthy effects. In this sense, the importance of fiber in the complex matrix of relationships between nutrients lies in its capacity to facilitate interaction among food components $[27,30]$.

Regarding the minor food groups that contribute to total fiber intake, studies on food consumption commonly include as sources of fiber and nutrients groups like appetizers, ready-to-eat meals, and sauces and condiments $[39,56,57]$. These food groups are usually consumed by the studied population, arguably due to reasons such as trends, price or lack of time for cooking. The relationship between numerous foods belonging to these groups and chronic diseases linked to economic development such as gastrointestinal diseases, obesity and type 2 diabetes, among others, is well established $[13,90]$. Fiber-enriched snacks also often contain a higher percentage of energy from carbohydrates and added sugars [36]. Thus, promoting improved patterns of fiber intake regardless of the fiber source could have counterproductive effects $[89,90]$.

While considering the food groups that most contribute to fiber intake (legumes, cereals, fruit and vegetables) the students' behavior is similar, when we analyze the groups that contribute less fiber, significant differences appear. The high fiber intake from these products among the American students contrasts with the absence of intake from sauces and precooked meals among their Tunisian counterparts. Furthermore, the idea that the beneficial properties of fiber may be isolated from intrinsically healthy foods, and added as ingredients to other foods perceived to be less healthy, is not always feasible, since the matrix of intact fiber may be affected during processing and, consequently, its healthy properties could be modified [27].

It should also be taken into account that even canned food with high levels of fiber such as legumes contain unhealthy ingredients not used in culinary preparation [16,91]. This could be the case of the students from FIU. Consumption of canned beans showed a major impact on fiber intake differences between the lowest and the highest American fiber consumers. In Spanish students, this increase was mainly due to the differences in fiber consumption from cereals. In contrast, differences between the lowest and the highest Tunisian fiber consumers were mainly accounted for by the differences in fiber consumption in the fruit group, it being important to note that most fruit consumed in Tunisia is natural whole fruit. 


\subsection{Third Level}

The pattern of fiber intake among young adults with a high level of education reflects the impact of globalization and cultural factors in their countries of origin, both in the amount of fiber consumed and the main food sources of fiber. Low levels of fiber intake were observed and none of the countries reached recommended levels. However, high-income countries are more aware of the need to increase fiber intake in order to improve health. In these countries, a large variety of cheap and easy to eat fiber-enriched food, much appreciated by young adults, is available (snacks, bran breakfast cereals) [36,92]. This fact could clearly contribute to enhanced fiber intake. Furthermore, when the groups of appetizers, ready-to-eat meals, and sauces were analyzed, the large differences found between participants reflect the influence of countries' different food environments even in a population as homogeneous as young adults with a high level of education.

Among chronic diseases associated with economic development, obesity is one of the most serious public health problems [70], and fiber is associated with satiety [26,27]. Therefore, the relationship between fiber intake and excess weight is of great interest $[34,55,85]$, especially since we know that younger adults are now gaining weight at a faster rate than the generation of their parents [93]. In this sense, our work confirms the effect of economic development on BMI since the prevalence of people with a BMI above the normal range (obese and overweight) was significantly lower in Tunisian participants than in American ones.

Taking into account the increase of pandemic chronic diseases and deaths caused by cancer and the difficulty of finding biomarkers for fiber intake [94,95], this study could provide a useful opportunity to reflect on the consequences of economic development and an incorrect interpretation of guidelines [96]. In line with other studies $[19,27]$, this survey stresses the need to redefine the concept of fiber, and to further study the health effects of fiber intake from different food sources. Therefore, more studies are needed in different populations, including young adults, given the importance for the future of improving habits during this life stage.

Finally, it should be noted that a strength of this study is the homogeneity in the data collection process. A small, qualified team collected data in the three countries. The entire process was developed in consultation and coordination with experts from each University, who also advised on specific issues in each country. The high educational level of our population guarantees the quality and validity of the self-reported information. However, some limitations of this study are those inherent to the use of 24-h recalls. Omission of some food intakes (such as snacks or side-dishes) and inaccuracy of estimation of portion size are potential sources of error when $24 \mathrm{~h}$ recalls are used. Moreover, other factors such as season may contribute to daily variation in nutrient intakes which is largely depending on cultural and food availability. In low-middle income countries seasonal effects are relatively strong. This could be the case of Tunisia where data were collected in winter, so fruit and vegetables rich in fiber are more scarce. Therefore actual fiber consumption could be higher than results obtained in this analysis. In the USA and Spain, with extensive food preservation and transportation systems, seasonal effects are relative weaker.

On the other hand, in each country, students from a specific university are going to have a different cultural and ethnic background than in other parts of the country. Therefore, their dietary intake could differ from students in other areas of the country or the general population.

\section{Conclusions}

In this work, none of the countries studied reached recommended levels of fiber intake. According to both the current definition of dietary fiber and the established adequate intakes, the conclusion of this work is that the diet quality determined by fiber intake of students from FIU is higher than that of Mediterranean students (UCA and UCLM). This finding, however, does not coincide with the classic hypothesis about the impact of economic development on fiber intake: "economic development is linked to a decrease in fiber intake". Total fiber intake increased with energy intake but did not depend on smoking habits and physical activity in any country. 
Regardless of country, the main food groups that contributed fiber to the diet were cereals, legumes, vegetables and fruits. The cereal group provided the largest amount of fiber in the three countries, but while vegetables were the second group providing fiber among Spanish and Tunisian participants, fruit was the second group among American participants.

Although fiber intake showed great homogeneity among participants regarding the main fiber food groups, differences were found when appetizers, ready-to-eat meals, and sauces and condiments were analyzed. Students from FIU obtained almost a fifth of their total fiber intake from these groups, which is twice the percentage for students from UCLM and seven times that among students from UCA.

The conclusions of this work apply to the Universities studied in each country. However, the limitations of the study do not let us extrapolate the results to the countries as a whole.

Supplementary Materials: The following are available online at www.mdpi.com/2072-6643/9/9/1030/s1, Tables S1-S3: Sociodemographic and lifestyle characteristics of the sample by countries (Spain, Tunisia, and the USA), Tables S4-S6: Total fiber intake (g) by countries (Spain, Tunisia, and the USA), and Table S7: Appetizers, prepared and precooked meals, and sauces and condiments consumed by the studied population.

Acknowledgments: The authors are very grateful to Fatman Huffman, Chair of the Department of Dietetics and Nutrition, Robert Stempel College of Public Health and Social Work (FIU) for her comprehensive and kind assistance. Thanks to Adriana Campa and Thouraya Daouas for their advice in data collection protocol at FIU and UCA, respectively. Authors also thank Jacqueline Hernández, Faustino Cervera, and Cruz Vico for their support and cooperation in data collection. This research was supported by the University of Castilla-La Mancha, Spain (Support for I+D+I Research Groups: GI20153153, GI20163500, and GI20174031).

Author Contributions: María José García-Meseguer designed and conducted the research. Amalia Delicado-Soria and Ramón Serrano-Urrea analyzed data. María José García-Meseguer and Ramón Serrano-Urrea wrote the paper. Amalia Delicado-Soria designed tables and figures. All authors participated in the discussion, and read and approved the final version of the manuscript.

Conflicts of Interest: The authors have no financial or any other kind of personal conflicts with this paper.

\section{References}

1. Burkitt, D.P.; Walker, A.R.; Painter, N.S. Effect of dietary fibre on stools and the transit-times, and its role in the causation of disease. Lancet 1972, 2, 1408-1412. [CrossRef]

2. Trowell, H. Why a new term for dietary fiber? Am. J. Clin. Nutr. 1977, 30, 1003-1004. [PubMed]

3. Slavin, J.L. Dietary fiber: Classification, chemical analyses, and food sources. J. Am. Diet. Assoc. 1987, 87, 1164-1171. [CrossRef] [PubMed]

4. Anderson, J.W.; Smith, B.M.; Gustafson, N.J. Health benefits and practical aspects of high-fiber diets. Am. J. Clin. Nutr. 1994, 59, 1242S-1247S. [PubMed]

5. Marlett, J.A.; McBurney, M.I.; Slavin, J.L. Position of the American Dietetic Association: Health implications of dietary fiber. J. Am. Diet. Assoc. 2002, 102, 993-1000. [CrossRef]

6. Bonnema, A.L.; Kolberg, L.W.; Thomas, W.; Slavin, J.L. Gastrointestinal tolerance of chicory inulin products. J. Am. Diet. Assoc. 2010, 110, 865-868. [CrossRef] [PubMed]

7. Yang, J.; Wang, H.P.; Zhou, L.; Xu, C.F. Effect of dietary fiber on constipation: A Meta Analysis. World J. Gastroenterol. 2012, 18, 7378-7383. [CrossRef] [PubMed]

8. Grosso, G.; Bella, F.; Godos, J.; Sciacca, S.; Del Rio, D.; Ray, S.; Galvano, F.; Giovannucci, E.L. Possible role of diet in cancer: Systematic review and multiple meta-analyses of dietary patterns, lifestyle factors, and cancer risk. Nutr. Rev. 2017, 75, 405-419. [CrossRef]

9. Meyer, K.A.; Kushi, L.H.; Jacobs, D.R., Jr.; Slavin, J.; Sellers, T.A.; Folsom, A.R. Carbohydrates, dietary fiber, and incident type 2 diabetes in older women. Am. J. Clin. Nutr. 2000, 71, 921-930. [PubMed]

10. Ye, E.Q.; Chacko, S.A.; Chou, E.L.; Kugizaki, M.; Liu, S. Greater whole-grain intake is associated with lower risk of type 2 diabetes, cardiovascular disease, and weight gain. J. Nutr. 2012, 142, 1304-1413. [CrossRef] [PubMed]

11. Cho, S.S.; Qi, L.; Fahey, G.C., Jr.; Klurfeld, D.M. Consumption of cereal fiber, mixtures of whole grains and bran, and whole grains and risk reduction in type 2 diabetes, obesity, and cardiovascular disease. Am. J. Clin. Nutr. 2013, 98, 594-619. [CrossRef] [PubMed] 
12. Buil-Cosiales, P.; Zazpe, I.; Toledo, E.; Corella, D.; Salas-Salvadó, J.; Diez-Espino, J.; Ros, E.; Fernandez-Creuet Navajas, J.; Santos-Lozano, J.M.; Arós, F.; et al. Fiber intake and all-cause mortality in the Prevención con Dieta Mediterránea (PREDIMED) study. Am. J. Clin. Nutr. 2014, 100, 1498-1507. [CrossRef] [PubMed]

13. Allman-Farinelli, M.; Partridge, S.R.; Roy, R. Weight-Related Dietary Behaviors in Young Adults. Curr. Obes. Rep. 2016, 5, 23-29. [CrossRef] [PubMed]

14. World Health Organization (WHO). Diet, Nutrition, and the Prevention of Chronic Diseases; Report of a WHO Study Group; WHO Technical Report Series 797; WHO Library Cataloguing-in-Publication Data: Geneva, Switzerland, 1990; Available online: http:/ /www.who.int/nutrition/publications/obesity/WHO_ TRS_797/en/ (accessed on 3 June 2017).

15. Nishida, C.; Uauy, R.; Kumanyika, S.; Shetty, P. The joint WHO/FAO expert consultation on diet, nutrition and the prevention of chronic diseases: Process, product and policy implications. Public Health Nutr. 2004, 7, 245-250. [CrossRef] [PubMed]

16. Freedman, M.R.; Fulgoni, V.L., III. Canned vegetables and fruit consumption is associated with changes in nutrient intake and higher diet quality in children and adults: National Health and Nutrition Examination Survey 2001-2010. J. Acad. Nutr. Diet. 2016, 116, 940-948. [CrossRef] [PubMed]

17. Howlett, J.F.; Betteridge, V.A.; Champ, M.; Craig, S.A.; Meheust, A.; Jones, J.M. The definition of dietary fiber-Discussions at the Ninth Vahouny Fiber Symposium: Building scientific agreement. Food Nutr. Res. 2010, 54, 5750. [CrossRef] [PubMed]

18. Jones, J.M. Dietary fiber future directions: Integrating new definitions and findings to inform nutrition research and communication. Adv. Nutr. 2013, 4, 8-15. [CrossRef] [PubMed]

19. Fuller, S.; Beck, E.; Salman, H.; Tapsell, L. New horizons for the study of dietary fiber and health: A review. Plant Foods Hum. Nutr. 2016, 71, 1-12. [CrossRef] [PubMed]

20. Institute of Medicine of the National Academies (IoMa). Dietary, functional and total fiber. In Dietary Reference Intakes for Energy, Carbohydrate, Fiber, Fat, Fatty Acids, Cholesterol, Protein, and Amino Acids; The National Academies Press: Washington, DC, USA, 2005; pp. 339-421. Available online: https:/ /www.nap.edu/read/ 10490/ chapter/1 (accessed on 3 June 2017).

21. Slavin, J.L.; Savarino, V.; Parades-Diaz, A.; Fotopoulos, G.A. Review of the role of soluble fiber in health with specific reference to wheat dextrin. J. Int. Med. Res. 2009, 37, 1-17. [CrossRef] [PubMed]

22. Lattimer, J.M.; Haub, M.D. Effects of dietary fiber and its components on metabolic health. Nutrients 2010, 2, 1266-1289. [CrossRef] [PubMed]

23. Brownawell, A.M.; Caers, W.; Gibson, G.R.; Kendall, C.W.; Lewis, K.D.; Ringel, Y.; Slavin, J.L. Prebiotics and the health benefits of fiber: Current regulatory status, future research, and goals. J. Nutr. 2012, 142, 962-974. [CrossRef] [PubMed]

24. Sanders, M.E.; Lenoir-Wijnkoop, I.; Salminen, S.; Merenstein, D.J.; Gibson, G.R.; Petschow, B.W.; Nieuwdorp, M.; Tancredi, D.J.; Cifelli, C.J.; Jacques, P.; et al. Probiotics and prebiotics: Prospects for public health and nutritional recommendations. Ann. N. Y. Acad. Sci. 2014, 1309, 19-29. [CrossRef] [PubMed]

25. Dahl, W.J.; Lockert, E.A.; Cammer, A.L.; Whiting, S.J. Effects of flax fiber on laxation and glycemic response in healthy volunteers. J. Med. Food 2005, 8, 508-511. [CrossRef] [PubMed]

26. Slavin, J.; Green, H. Dietary fibre and satiety. Nutr. Bull. 2007, 32, S32-S42. [CrossRef]

27. Wahlqvist, M.L. Food structure is critical for optimal health. Food Funct. 2016, 7, 1245-1250. [CrossRef] [PubMed]

28. Liu, R.H. Health benefits of fruit and vegetables are from additive and synergistic combinations of phytochemicals. Am. J. Clin. Nutr. 2003, 78, 517S-520S. [PubMed]

29. Okarter, N.; Liu, R.H. Health benefits of whole grain phytochemicals. Crit. Rev. Food Sci. Nutr. 2010, 50, 193-208. [CrossRef] [PubMed]

30. Jones, J.M. Dietary fibre's Co-Passengers: Is it the fiber or the co-passengers? In Dietary Fiber: New Frontiers for Food and Health; Wageningen Academic Publishers: Wageningen, The Netherlands, 2010; pp. 365-378. [CrossRef]

31. Saura-Calixto, F. Dietary fiber as a carrier of dietary antioxidants: An essential physiological function. J. Agric. Food Chem. 2011, 59, 43-49. [CrossRef] [PubMed]

32. De Vries, J.; Birkett, A.; Hulshof, T.; Verbeke, K.; Gibes, K. Effects of cereal, fruit and vegetable fibers on human fecal weight and transit time: A comprehensive review of intervention trials. Nutrients 2016, 8, 130. [CrossRef] [PubMed] 
33. Buil-Cosiales, P.; Martinez-Gonzalez, M.A.; Ruiz-Canela, M.; Díez-Espino, J.; García-Arellano, A.; Toledo, E. Consumption of fruit or fiber-fruit decreases the risk of cardiovascular disease in a Mediterranean young cohort. Nutrients 2017, 9, 295. [CrossRef] [PubMed]

34. González-Rodríguez, L.; Perea Sánchez, J.M.; Aranceta-Bartrina, J.A.; González-Gross, M.; Serra-Majem, L.; Varela-Moreiras, G.; Ortega, R.M. Intake and Dietary Food Sources of Fibre in Spain: Differences with Regard to the Prevalence of Excess Body Weight and Abdominal Obesity in Adults of the ANIBES Study. Nutrients 2017, 9, 326. [CrossRef] [PubMed]

35. Howarth, N.C.; Huang, T.T.K.; Roberts, S.B.; McCrory, M.A. Dietary Fiber and Fat Are Associated with Excess Weight in Young and Middle-Aged US Adults. J. Am. Diet. Assoc. 2005, 105, 1365-1372. [CrossRef] [PubMed]

36. Myhre, J.; Løken, E.; Wandel, M.; Andersen, L. The contribution of snacks to dietary intake and their association with eating location among Norwegian adults-Results from a cross-sectional dietary survey. BMC Public Health 2015, 15, 1-9. [CrossRef] [PubMed]

37. Bielemann, R.M.; Motta, J.V.; Minten, G.C.; Horta, B.L.; Gigante, D.P. Consumption of ultra-processed foods and their impact on the diet of young adults. Rev. Saude Publica 2015, 49, 28. [CrossRef] [PubMed]

38. El Ansari, W.; Stock, C.; Mikolajczyk, R.T. Relationships between food consumption and living arrangements among university students in four European countries-A cross-sectional study. Nutr. J. 2012, 11, 28. [CrossRef] [PubMed]

39. García-Meseguer, M.J.; Cervera Burriel, F.; Vico García, C.; Serrano-Urrea, R. Adherence to Mediterranean diet in a Spanish university population. Appetite 2014, 78, 156-164. [CrossRef] [PubMed]

40. Sharma, B.; Harker, M.; Harker, D.; Reinhard, K. Younth transition to University in Germany and Australia. An empirical investigation of healthy eating behaviour. J. Youth Stud. 2010, 13, 353-367. [CrossRef]

41. Erlich, R.; Yngve, A.; Wahlqvist, M.L. Cooking as a healthy behaviour. Public Health Nutr. 2012, 15, 1139-1140. [CrossRef] [PubMed]

42. Szczuko, M.; Gutowska, I.; Seidler, T. Nutrition and nourishment status of Polish students in comparison with students from other countries. Rocz. Panstw. Zakl. Hig. 2015, 66, 261-268. [PubMed]

43. Ludwig, D.S.; Pereira, M.A.; Kroenke, C.H.; Hilner, J.E.; Van Horn, L.; Slattery, M.L.; Jacobs, D.R., Jr. Dietary fiber, weight gain, and cardiovascular disease risk factors in young adults. JAMA 1999, 282, 1539-1546. [CrossRef] [PubMed]

44. Farvid, M.S.; Eliassen, A.H.; Cho, E.; Liao, X.; Chen, W.Y.; Willett, W.C. Dietary fiber intake in young adults and breast cancer risk. Pediatrics 2016, 137, e20151226. [CrossRef] [PubMed]

45. Chourdakis, M.; Tzellos, T.; Papazisis, G.; Toulis, K.; Kouvelas, D. Eating habits, health attitudes and obesity indices among medical students in northern Greece. Appetite 2010, 55, 722-725. [CrossRef] [PubMed]

46. Parminder, K.G.; Charajeet, K.H. Intake of nutrients, phytin P, polyphenolic compounds, oxalates and dietary fibre by university campus residents. Ecol. Food Nutr. 1995, 34, 11-17. [CrossRef]

47. Nicklas, T.; Nicklas, T.A.; Farris, R.P.; Myers, L.; Berenson, G.S. Dietary fiber intake of children and young adults: The Bogalusa Heart Study. J. Am. Diet. Assoc. 1995, 95, 209-214. [CrossRef]

48. Lagerpusch, M.; Enderle, J.; Later, W.; Eggeling, B.; Pape, D.; Muller, M.J.; Bosy-Westphal, A. Impact of glycaemic index and dietary fibre on insulin sensitivity during the refeeding phase of a weight cycle in young healthy men. Br. J. Nutr. 2013, 109, 1606-1616. [CrossRef] [PubMed]

49. The World Bank. New Country Classifications by Income Level. Available online: https:/ /blogs.worldbank. org/opendata/new-country-classifications-2016 (accessed on 3 June 2017).

50. Mokhtar, N.; El Ati, J.; Chabir, R.; Bour, A.; Elkari, K.; Schlossman, N.P.; Caballero, B.; Aguenaou, H. Diet culture and obesity in northern Africa. J. Nutr. 2001, 131, 887S-892S. [PubMed]

51. World Health Organization (WHO), Regional Office for the Eastern Mediterranean. Promoting a Healthy Diet for the WHO Eastern Mediterranean Region: User-Friendly Guide; WHO: Cairo, Egypt, 2012. Available online: http:/ / applications.emro.who.int/dsaf/emropub_2011_1274.pdf?ua=1 (accessed on 3 June 2017).

52. Agencia Española de Seguridad Alimentaria y Nutrición (AESAN). Encuesta Nacional de Ingesta Dietética de la Población Española. (2009-2010) (ENIDE). 2012. Available online: http:/ / www.tappers.es/media/ wysiwyg/valoracion_nutricional_enide_macronutrientes.pdf (accessed on 3 June 2017).

53. U.S. Department of Agriculture; U.S. Department of Health and Human Services. Dietary Guidelines for Americans, 7th ed.; U.S. Government Printing Office: Washington, DC, USA, 2010. Available online: https: / / health.gov / dietaryguidelines / dga2010/dietaryguidelines2010.pdf (accessed on 3 June 2017). 
54. Hoy, M.K.; Goldman, J.D. Fiber Intake of the U.S. Population: What We Eat in America, NHANES 2009-2010. Food Surveys Research Group Dietary Data Brief No. 12. U.S. Department of Agriculture, 2014. Available online: https:/ /www.ars.usda.gov/ARSUserFiles/80400530/pdf/DBrief/12_fiber_intake_ 0910.pdf (accessed on 3 June 2017).

55. Aounallah-Skhiri, H.; Traissac, P.; El Ati, J.; Eymard-Duvernay, S.; Landais, E.; Achour, N.; Delpeuch, F.; Ben Romdhane, H.; Maire, B. Nutrition transition among adolescents of a south-Mediterranean country: Dietary patterns, association with socio-economic factors, overweight and blood pressure. A cross-sectional study in Tunisia. Nutr. J. 2011, 10, 38. [CrossRef] [PubMed]

56. Ruiz, E.; Ávila, J.M.; Valero, T.; Del Pozo, S.; Rodriguez, P.; Aranceta-Bartrina, J.; Gil, Á.; González-Gross, M.; Ortega, R.M.; Serra-Majem, L.; et al. Macronutrient distribution and dietary sources in the Spanish population: Findings from the ANIBES study. Nutrients 2016, 8, 177. [CrossRef] [PubMed]

57. McGill, C.R.; Devareddy, L. Ten-year trends in fiber and whole grain intakes and food sources for the United States population: National Health and Nutrition Examination Survey 2001-2010. Nutrients 2015, 7, 1119-1130. [CrossRef] [PubMed]

58. Willet, W. Issues in Analysis and Presentation of Dietary Data. Nutritional Epidemiology, 3rd ed.; Oxford University Press: New York, NY, USA, 2013.

59. World Health Organization (WHO). Obesity: Preventing and Managing the Global Epidemic; Report of a WHO Consultation; WHO Technical Report Series 894; WHO Library Cataloguing-in-Publication Data: Geneva, Switzerland, 2000; Available online: http:/ / www.who.int/nutrition/publications/obesity/WHO_TRS_894/ en/ (accessed on 3 June 2017).

60. Gómez, C.; Loria, V.; Lourenço, T. Guía Visual de Alimentos y Raciones; EDIMSA (Editores Médicos, S.A.): Madrid, España, 2007.

61. WebMD Health Corporation. The WebMD Portion Size Plate. Available online: http://www.webmd.com/ diet/healthtool-portion-size-plate (accessed on 15 May 2017).

62. Institute of Medicine of the National Academies (IoMb). Physical activity. In Dietary Reference Intakes for Energy, Carbohydrate, Fiber, Fat, Fatty Acids, Cholesterol, Protein, and Amino Acids; The National Academies Press: Washington, DC, USA, 2005; pp. 880-935. Available online: https:/ /www.nap.edu/read/10490/chapter/12 (accessed on 15 May 2017).

63. Institut National de Nutrition \& de Technologie Alimentaire (INNTA). Table de Composition des Aliments Tunisiens. Available online: http:/ / www.institutdenutrition.rns.tn/ (accessed on 15 May 2017).

64. Moreiras, O.; Carbajal, A.; Cabrera, L.; Cuadrado, C. Tablas de Composición de Alimentos. Guía de Prácticas, 17th ed.; Ediciones Pirámide: Madrid, España, 2015.

65. U.S. Department of Agriculture. USDA Food Composition Databases. Available online: https://ndb.nal. usda.gov/ndb/ (accessed on 15 May 2017).

66. Vellas, B.; Guigoz, Y.; Garry, P.J.; Nourhashemi, F.; Bennahum, D.; Lauque, S.; Albarede, J.L. The Mini Nutritional Assessment (MNA) and its use in grading the nutritional state of elderly patients. Nutrition 1999, 15, 116-122. [CrossRef]

67. Tur, J.A.; Serra-Majem, L.; Romaguera, D.; Pons, A. Does the diet of the Balearic population, a Mediterranean type diet, still provide adequate antioxidant nutrient intakes? Eur. J. Nutr. 2005, 44, 204-213. [CrossRef] [PubMed]

68. Wu, L.; Wang, Z.; Zhu, J.; Murad, A.L.; Prokop, L.J.; Murad, M.H. Nut consumption and risk of cancer and type 2 diabetes: A systematic review and meta-analysis. Nutr. Rev. 2015, 73, 409-425. [CrossRef] [PubMed]

69. Omran, A.R. The epidemiologic transition: A theory of the epidemiology of population change. Milbank $Q$. 2005, 83, 731-757. [CrossRef] [PubMed]

70. Popkin, B.M.; Adair, L.S.; Ng, S.W. Global nutrition transition and the pandemic of obesity in developing countries. Nutr. Rev. 2012, 70, 3-21. [CrossRef] [PubMed]

71. Dahl, W.J.; Stewart, M.L. Position of the Academy of Nutrition and Dietetics: Health implications of dietary fiber. J. Acad. Nutr. Diet. 2015, 115, 1861-1870. [CrossRef] [PubMed]

72. Wardle, J.; Haase, A.M.; Steptoe, A. Body image and weight control in young adults: International comparisons in university students from 22 countries. Inter. J. obes. 2006, 30, 644-651. [CrossRef] [PubMed]

73. Slavin, J. Fiber and prebiotics: Mechanisms and health benefits. Nutrients 2013, 22, 1417-1435. [CrossRef] [PubMed] 
74. Xu, Z.; Knight, R. Dietary effects on human gut microbiome diversity. Br. J. Nutr. 2015, 113, S1-S5. [CrossRef] [PubMed]

75. Cervera Burriel, F.; Serrano Urrea, R.; Daouas, T.; Delicado Soria, A.; García Meseguer, M.J. Food habits and nutritional assessment in a Tunisian university population. Nutr. Hosp. 2014, 30, 1350-1358. [CrossRef] [PubMed]

76. Liu, Y.; Colditz, G.A.; Cotterchio, M.; Boucher, B.A.; Kreiger, N. Adolescent dietary fiber, vegetable fat, vegetable protein, and nut intakes and breast cancer risk. Breast Cancer Res. Treat. 2014, 145, 461-470. [CrossRef] [PubMed]

77. Larsson, S.C.; Wolk, A. Dietary fiber intake is inversely associated with stroke incidence in healthy Swedish adults. J. Nutr. 2014, 144, 1952-1955. [CrossRef] [PubMed]

78. Mrabet, A.; Rodríguez-Arcos, R.; Guillén-Bejarano, R.; Chaira, N.; Ferchichi, A.; Jiménez-Araujo, A. Dietary fiber from Tunisian common date cultivars (Phoenix dactylifera L.): Chemical composition, functional properties, and antioxidant capacity. J. Agric. Food Chem. 2012, 60, 3658-3664. [CrossRef] [PubMed]

79. Eurostat. GDP at Regional Level. Available online: http://ec.europa.eu/eurostat/statistics-explained/ index.php/GDP_at_regional_level/es (accessed on 25 May 2017).

80. Food Agriculture Organization (FAO). Tunisia Case Study. Prepared for FAO as part of the State of the World's Forests 2016 (SOFO). 2015. Available online: http:/ / www.fao.org/3/a-c0185e.pdf (accessed on 25 May 2017).

81. World Health Organization (WHO)/Food Agriculture Organization (FAO). Global and regional food consumption patterns and trends. In Diet, Nutrition and the Prevention of Chronic Diseases; Report of a Joint WHO/FAO Expert Consultation; WHO Technical Report Series 916; WHO Library Cataloguing-in-Publication Data: Geneva, Switzerland, 2003; pp. 13-29. Available online: ftp:/ /ftp.fao.org/ docrep/fao/005/ac911e/ac911e01.pdf (accessed on 25 May 2017).

82. Florence, M.D.; Asbridge, M.; Veugelers, P.J. Diet quality and academic performance. J. Sch. Health 2008, 78, 209-215. [CrossRef] [PubMed]

83. Food Agriculture Organization (FAO). Profil Nutritionnel de la Tunisie. Division de l'Alimentation et de la Nutrition. 2005. Available online: http:/ / www.fao.org/docrep/017/ap850f/ap850f.pdf (accessed on 25 May 2017).

84. Chourdakis, M.; Tzellos, T.; Pourzitaki, C.; Toulis, K.A.; Papazisis, G.; Kouvelas, D. Evaluation of dietary habits and assessment of cardiovascular disease risk factors among Greek university students. Appetite 2011, 57,377-383. [CrossRef] [PubMed]

85. Du, H.; van der A, D.L.; Boshuizen, H.C.; Forouhi, N.G.; Wareham, N.J.; Halkjaer, J.; Tjønneland, A.; Overvad, K.; Jakobsen, M.U.; Boeing, H.; et al. Dietary fiber and subsequent changes in body weight and waist circumference in European men and women. Am. J. Clin. Nutr. 2010, 91, 329-336. [CrossRef] [PubMed]

86. Ben Slama, F.; Jebali, N.; Chemli, R.; Ben Rayana, C.; Achour, A.; Najar, M.F.; Achour, N.; Belhadj, O. Dietary fiber in the diets of urban Tunisian women: Association of fiber intake with BMI, waist circumference and blood chemistry: Preliminary study. Clin. Res. Hepatol. Gastroenterol. 2011, 35, 750-754. [CrossRef] [PubMed]

87. Sawada, N.; Iwasaki, M.; Yamaji, T.; Shimazu, T.; Sasazuki, S.; Inoue, M.; Tsugane, S.; Japan Public Health Center-based Prospective Study Group. Fiber intake and risk of subsequent prostate cancer in Japanese men. Am. J. Clin. Nutr. 2015, 101, 118-125. [CrossRef] [PubMed]

88. Gordon, T.; Fisher, M.; Rifkind, B.M. Some difficulties inherent in the interpretation of dietary data from free-living populations. Am. J. Clin. Nutr. 1984, 39, 152-156. [PubMed]

89. Lesser, L.I.; Mazza, M.C.; Lucan, S.C. Nutrition myth and healthy dietary advice in clinical practice. Am. Fam. Physician 2015, 91, 634-638. [PubMed]

90. Shau, J.P.; Chen, P.H.; Chan, C.F.; Hsu, Y.; Wu, T.C.; James, F.E.; Pan, W.H. Fast foods-Are they a risk factor for functional gastrointestinal disorders? Asia Pac. J. Clin. Nutr. 2016, 25, 393-401. [CrossRef] [PubMed]

91. US Food \& Drug Administration. CFR-Code of Federal Regulations Title 21. Available online: https: / / www.accessdata.fda.gov/scripts/cdrh/cfdocs/cfcfr/CFRSearch.cfm?fr=172.120 (accessed on 3 June 2017).

92. Trude, A.C.; Kharmats, A.Y.; Hurley, K.M.; Anderson Steeves, E.; Talegawkar, S.A.; Gittelsohn, J. Household, psychosocial, and individual-level factors associated with fruit, vegetable, and fiber intake among low-income urban African American youth. BMC Public Health 2016, 16, 872. [CrossRef] [PubMed] 
93. Allman-Farinelli, M.A.; Chey, T.; Bauman, A.E.; Gill, T.; James, W.P. Age, period and birth cohort effects on prevalence of overweight and obesity in Australian adults from 1990 to 2000. Eur. J Clin. Nutr. 2008, 62, 898-907. [CrossRef] [PubMed]

94. McKeown-Eyssen, G.E.; Yeung, K.S.; Bright-See, E. Assessment of past diet in epidemiologic studies. Am. J. Epidemiol. 1986, 124, 94-103. [CrossRef] [PubMed]

95. Weitkunat, K.; Schumann, S.; Nickel, D.; Hornemann, S.; Petzke, K.J.; Schulze, M.B.; Pfeiffer, A.F.; Klaus, S. Odd-chain fatty acids as a biomarker for dietary fiber intake: A novel pathway for endogenous production from propionate. Am. J. Clin. Nutr. 2017, 105, 1544-1551. [CrossRef] [PubMed]

96. Kranz, S.; Dodd, K.W.; Juan, W.Y.; Johnson, L.K.; Jahns, L. Whole grains contribute only a small proportion of dietary fiber to the U.S. diet. Nutrients 2017, 9, 153. [CrossRef] [PubMed]

2017 by the authors. Licensee MDPI, Basel, Switzerland. This article is an open access article distributed under the terms and conditions of the Creative Commons Attribution (CC BY) license (http:// creativecommons.org/licenses/by/4.0/). 